\title{
ON THE THEORY OF INTEGRAL EQUATIONS WITH DISCONTINUOUS KERNELS*
}

\author{
BY \\ RUDOLPH E. LANGER
}

\section{Chapter 1. Introduction}

This paper is concerned with the theory of the integral equation whose salient characteristic is that its kernel $K(x, \xi)$ is discontinuous along the line $\xi=x$. It is closely related with the theory of the integral equation characterized by discontinuities in the partial derivatives of the kernel for $\xi=x$, of which more specific mention is made below.

The point of departure for the consideration involved is in each case furnished by the familiar fact of the equivalence of a differential system composed of an equation

$$
p_{0} \frac{d^{n} u}{d x^{n}}+\cdots+p_{n} u=\lambda u,
$$

and homogeneous boundary conditions, with the integral equation

$$
u(x)=\lambda \int_{a}^{b} G(x, \xi) u(\xi) d \xi,
$$

in which the kernel is the Green's function of the reduced differential system. The existing theory of differential boundary problems guarantees the existence of characteristic values of $\lambda$ and corresponding functions $u(x)$ for the differential system in a broad class of cases. The same is true, therefore, for the equivalent integral equation, and viewed from the standpoint of the theory of integral equations these facts must be attributable to the peculiarities of the Green's function which serves as a kernel.

Fixing the attention for the moment on the case in which the differential system is of the second order and self-adjoint, the properties of the kernel which are of particular interest in this connection are (1), its symmetry in its arguments, and (2), the finite non-vanishing discontinuities in its first partial derivatives for $\xi=x$. The extent to which symmetry of the kernel serves as a basis for a theory of integral equations is shown by the theory of Hilbert and Schmidt. The question of the extent to which the second

\footnotetext{
* Presented to the Society, January 1, 1926; received by the editors January 21 and March
} 26, 1926. 
property mentioned serves as such a basis has remained open. It appears to have been touched upon first by Birkhoff in 1906 in an unpublished discussion of the equation with kernel both symmetric and discontinuous.*

The development of a theory based only on the discontinuity of the first partial derivatives of the kernel was taken up by Mrs. Eleanor P. Brown in a thesis prepared under the direction of Birkhoff and presented at Radcliffe College in 1921. This theory will be presented shortly in a joint paper by Mrs. Brown and the author.

If the differential system under consideration is of the first order the discontinuity along the line $\xi=x$ occurs in the Green's function itself. It is this property which is taken as the distinguishing feature of the kernel of the integral equation to which the present paper is devoted. While the analytic details which are of interest differ in many respects, the methods used here are in the main parallel to those of the paper referred to above which is to embody Mrs. Brown's thesis. They are similar also in many respects to the methods employed by Birkhoff and the author in a treatment of differential boundary problems. $\ddagger$

The content of the paper may be roughly summarized as follows. In Chapter 2 the hypotheses on the kernel of the given equation are enunciated and a change of variables is deduced which serves to normalize the discontinuities in the kernel and its first partial derivatives. In Chapter 3 the integral equation is transformed into a system composed of an integrodifferential equation and a boundary condition, and in Chapter 4 the equivalence of this system with the given equation is established. Chapters 5, 6, and 7 are concerned with the integro-differential equation alone (without the boundary condition), and it is shown that the equation is possessed of a solution for all values of the parameter which are sufficiently large. The asymptotic form of this solution in any right-hand or left-hand half-plane is there deduced.

In Chapter 8 the boundary condition is introduced and by means of it the existence, under certain restrictions, of infinitely many characteristic values for the parameter is proved and the asymptotic form of these values is obtained. In Chapters 9, 10, and 11, the normalized asymptotic forms of the characteristic functions for the given integral equation and its associated

* Bulletin of the American Mathematical Society, vol. 13 (1906), p. 62.

$\dagger$ Formerly Miss Eleanor Pairman.

† G. D. Birkhoff and R. E. Langer, Boundary problems and developments associated with a system of ordinary linear differential equations of the first order, Proceedings of the American Academy of Arts and Sciences, vol. 58, pp. 51-128; April, 1923 
equation are developed, and it is proved that when the given equation has only simple characteristic values the set of characteristic functions is closed. Lastly in Chapter 12 the expansion of an arbitrary function in a series of the solutions is considered. It is shown that for the expansion of any function $f(x)$ which is integrable in the sense of Lebesgue the solutions of the integral equation have essentially the same properties as the solutions of a related system composed of a differential equation of the first order and a homogeneous boundary condition. At the end points of the interval, and only there, do the two expansions behave in dissimilar fashion. The related differential system in question here is a special case of a system previously studied by the author.*

In the appendix a simple example of an integral equation to which the developed theory applies is given, and by computed results various features of the theory are illustrated.

Chapter 2. The normalization of the equation

1. The given equation. We consider given the equation

$$
y(t)=\rho \int_{\alpha}^{\beta} \Gamma(t, \tau) y(\tau) d \tau,
$$

in which $\rho$ is a complex parameter. The kernel is real and satisfies the following conditions:

(i) that it is possessed of partial derivatives to those of order $n \geqq 1$ inclusive, these partial derivatives being continuous in the open regions

$$
R^{\prime}\left\{\begin{array} { l } 
{ \alpha \leqq \tau < t } \\
{ \alpha \leqq t \leqq \beta }
\end{array} \text { and } \quad R ^ { \prime \prime } \left\{\begin{array}{l}
t<\tau \leqq \beta \\
\alpha \leqq t \leqq \beta
\end{array}\right.\right.
$$

and approaching in each of these regions a finite limiting value at every point of the boundary $\tau=t$;

(ii) that

$$
\Gamma(t, \tau)]_{\tau=t-}^{\tau-t+}=\varphi(t) \neq 0 .
$$

2. The derivatives of $\varphi(t)$. The differentiability of the function $\varphi(t)$ defined in (ii) may be derived from the facts which we formulate as follows. 155-172.

- Developments associated with a boundary problem, these Trans actions, vol. 25 (1923), pp. 
LeMma. If $F(t, \tau)$ is any function possessed of the properties (i) above, then

$$
\begin{aligned}
& \frac{d F(t, t+)}{d t}=F_{t}(t, t+)+F_{\tau}(t, t+), \\
& \frac{d F(t, t-)}{d t}=F_{t}(t, t-)+F_{\tau}(t, t-) .
\end{aligned}
$$

Let $t_{2}>t_{1}$ be any two points of the interval $(\alpha, \beta)$. Then

$$
\frac{F\left(t_{2}, t_{2}+\right)-F\left(t_{1}, t_{1}+\right)}{t_{2}-t_{1}}=\frac{1}{\Delta t}\left\{F\left(t_{2}, t_{2}+\right)-F\left(t_{1}, t_{2}\right)+F\left(t_{1}, t_{2}\right)-F\left(t_{1}, t_{1}+\right)\right\},
$$

where $\Delta t=t_{2}-t_{1}$. By the law of the mean the right hand member of this equals

$$
F_{t}\left(t_{2}-\theta_{1} \Delta t, t_{2}\right)+F_{r}\left(t_{1}, t_{2}-\theta_{2} \Delta t\right), \quad 0<\theta_{i}<1 .
$$

Since this approaches the right hand member of (a) as a limit when either $t_{1}$ or $t_{2}$ is taken at $t$ and $\Delta t \rightarrow 0$ the proof of statement (a) is complete.

The proof of $(b)$ is precisely similar.

Since the kernel $\Gamma(t, \tau)$ and its partial derivatives to those of order $(n-1)$ are of the type of $F(t, \tau)$ of the lemma, we may differentiate the right hand member of the relation

$$
\varphi(t)=\Gamma(t, t+)-\Gamma(t, t-)
$$

$n$ times. This, together with the continuity of the resulting terms on the right, establishes the existence and continuity of $d^{n} \varphi(t) / d t^{n}$ on the interval $(\alpha, \beta)$.

3. The change of variables. By hypothesis the function $\varphi(t)$ maintains its sign on $(\alpha, \beta)$. With a proper distribution of the constant factors between $\rho$ and $\Gamma$ it will follow that $\varphi(t)>0$. We assume such a distribution. Then the continuous functions

$$
x=\int_{\alpha}^{t} \varphi(t) d t, \quad \xi=\int_{\alpha}^{\tau} \varphi(\tau) d \tau
$$

are monotonic increasing and may be used as new independent variables. Introducing them in equation (1) and denoting by $\bar{f}(x, \xi)$ the function into which $f(t, \tau)$ is transformed by the inverse of $(2)$, we obtain the form

$$
\bar{y}(x)=\lambda \int_{a}^{b} \Omega(x, \xi) \bar{y}(\xi) d \xi,
$$

in which 


$$
\begin{gathered}
\lambda=-\rho, \quad a=0, \quad b=\int_{\alpha}^{\beta} \varphi(\tau) d \tau, \\
\Omega(x, \xi)=-\frac{\bar{\Gamma}(x, \xi)}{\bar{\varphi}(\xi)} .
\end{gathered}
$$

It is readily verified that in the regions

$$
R_{1}\left\{\begin{array} { l } 
{ a \leqq \xi < x } \\
{ a \leqq x \leqq b }
\end{array} \quad \text { and } \quad R _ { 2 } \left\{\begin{array}{l}
x<\xi \leqq b \\
a \leqq x \leqq b
\end{array}\right.\right.
$$

and on the boundary $\xi=x, \Omega(x, \xi)$ is possessed of properties analogous to those under (i) above. Moreover

$$
\Omega(x, \xi)]_{\xi=x-}^{\xi=x+} \equiv-1 .
$$

Defining $\psi(x)$ now by the relation

$$
\left.\Omega_{x}(x, \xi)\right]_{\xi=x-}^{\xi=x+}=\psi(x),
$$

and introducing in (3) as a new dependent variable the function

$$
u(x)=\bar{y}(x) e^{\int_{a}^{x} \psi(x) d x},
$$

we obtain as the final form of the equation

$$
u(x)=\lambda \int_{a}^{b} K(x, \xi) u(\xi) d \xi
$$

where

$$
K(x, \xi)=\Omega(x, \xi) e^{\int_{\xi}^{x} \psi(x) d x} .
$$

This kernel $K(x, \xi)$ and its partial derivatives are undefined on the line $\xi=x$. We shall complete their definitions by designating them to have in the points of this line their limiting values as these points are approached in the region $R_{1}$.

4. The normalized equation. The equation (5) will be said to represent the normal form because of the following characteristics which its kernel is found to possess.

(A) The function $K(x, \xi)$ is possessed of partial derivatives to those of order $n \geqq 1$ inclusive, which are continuous in the region $R_{2}$ and in the closed region

$$
R_{1}^{\prime}\left\{\begin{array}{l}
a \leqq \xi \leqq x \\
a \leqq x \leqq b
\end{array}\right.
$$




$$
\begin{aligned}
& K(x, \xi)]_{\xi-x}^{\xi-x+} \equiv-1 ; \\
& \left.K_{x}(x, \xi)\right]_{\xi=x}^{\xi-x+} \equiv 0 .
\end{aligned}
$$

It may be observed that the differentiation of (B) and the application of (C) yield the further relation

$$
\left.K_{\xi}(x, \xi)\right]_{\xi=x}^{\xi-x+} \equiv 0 .
$$

THEOREM 1. Every integral equation of the type (1) with a kernel possessed of the properties (i) and (ii) above may, by a suitable change of variables, be transformed into an equation of the same type with a kernel possessed of the properties (A), (B), (C) and (D).

Chapter 3. Transformation of the integral EQUation into AN INTEGRO-DIFFERENTIAL SYSTEM

5. The auxiliary differential system. We consider in connection with the normalized equation the differential system

$$
\begin{gathered}
y^{\prime}(x)=0, \\
\mu y(a)+\nu y(b)=0 .
\end{gathered}
$$

The constants $\mu$ and $\nu$ are parameters, the choice of which is arbitrary, subject to restrictions to be imposed at certain points in the subsequent theory. In each case, however, only discrete values of the ratio $\mu / \nu$ will be excluded.

To begin, let $\mu$ and $\nu$ be chosen so that

$$
\mu / \nu \neq-1 \text {. }
$$

Then system (6) is incompatible and possesses a Green's function $G(x, \xi)$. This function and its derivatives are undefined on the line $\xi=x$ where $G(x, \xi)$ is discontinuous. We shall complete their definitions in the region $R$, composed of $R_{2}$ and $R_{1}^{\prime}$, by designating them to have in the points of the line $\xi=x$ their limiting values as these points are approached in the region $R_{1}$. We may then enumerate their characteristics as follows.

I. The functions $G(x, \xi), G_{x}(x, \xi)$, and $G_{\xi}(x, \xi)$ are continuous in $R_{\mathbf{2}}$ and $R_{1}^{\prime}$;

II.

$$
G(x, \xi)]_{\xi-x}^{\xi-x+} \equiv-1 ;
$$

III.

$$
G_{x}(x, \xi) \equiv G_{\xi}(x, \xi) \equiv 0 \text {; }
$$

IV.

$$
\mu G(a, \xi)+\nu G(b, \xi) \equiv 0 \text {; }
$$

V.

$$
\nu G(x, a)+\mu G(x, b) \equiv 0 \text {; }
$$


VI. the solution of the system

is given by

$$
\begin{gathered}
\omega^{\prime}(x)=f(x), \\
\mu \omega(a)+\nu \omega(b)=0
\end{gathered}
$$

$$
\omega(x)=\int_{a}^{b} G(x, t) f(t) d t ;
$$

VII.

$$
G(x, \xi)=-H(\xi, x),
$$

where $H(x, \xi)$ is the Green's function of the system adjoint to (6).

6. The construction of $K(x, \xi)$. It will be observed that $G(x, \xi)$ and the kernel $K(x, \xi)$ of the normalized equation (5), and their respective first partial derivatives, have the same discontinuities in the region $R$. We shall construct with the use of $K(x, \xi)$ another function $K(x, \xi)$ which maintains these characteristics, and in addition shares with $G(x, \xi)$ its properties IV and $\mathrm{V}$.

We assume now that for the given equation

(iii)

$$
n \geqq 2 \text {, }
$$

and that in the normalized form

$$
|K(a, b)|+|K(a, a+)+K(b, b)|+|K(b, a)| \neq 0 \text {. }
$$

Then $\mu$ and $\nu$ may be so chosen, subject to previous restriction, that

$$
\Delta=\mu^{2} K(a, b)+\mu \nu[K(a, a+)+K(b, b)]+\nu^{2} K(b, a) \neq 0 .
$$

We assume such a choice, and set

$$
\begin{aligned}
& \nu K(x, a)+\mu K(x, b)=W(x), \\
& \mu K(a, \xi)+\nu K(b, \xi)=V(\xi) .
\end{aligned}
$$

Clearly these functions $W(x)$ and $V(\xi)$ are possessed of continuous derivatives to those of order $n$ on the interval $(a, b)$. The function $K(x, \xi)$, defined by the formula

$$
K(x, \xi)=\frac{1}{\Delta}\left|\begin{array}{cc}
K(x, \xi) & W(x) \\
V(\xi) & \Delta
\end{array}\right|,
$$

is, therefore, found to possess the properties (A) to (D) of the kernel $K(x, \xi)$. From the relations

$$
\begin{aligned}
\mu W(a)+\nu W(b) & =\Delta, \\
\nu V(a)+\mu V(b) & =\Delta,
\end{aligned}
$$


it follows further that

$$
\begin{aligned}
\mu K(a, \xi)+\nu K(b, \xi) & \equiv 0, \\
\nu K(x, a)+\mu K(x, b) & \equiv 0 .
\end{aligned}
$$

7. The relation between $K(x, \xi)$ and $G(x, \xi)$. If $x$ is regarded for the moment as a parameter, the function

$$
\omega(x, \xi)=K(x, \xi)-G(x, \xi)
$$

is continuous together with its first derivative in $\xi$. Since by III, V, and (12) it is also a solution of the differential system

$$
\begin{aligned}
& \frac{\partial \omega(x, \xi)}{\partial \xi}=K_{\xi}(x, \xi), \\
& \nu \omega(x, a)+\mu \omega(x, b)=0,
\end{aligned}
$$

it is uniquely determined as such, and is by VI expressible in terms of the Green's function $H(x, \xi)$. Introducing $G(x, \xi)$ by virtue of VII, we may write therefore

$$
K(x, \xi)-G(x, \xi)=-\int_{a}^{b} K_{t}(x, t) G(t, \xi) d t .
$$

This relation, if $\xi$ is now looked upon as the parameter, is in form an integral equation for $G(x, \xi)$ as a function of $x$.

8. The solvability of the relation between $K(x, \xi)$ and $G(x, \xi)$. A sufficient condition for the solvability of equation (13) for $G(x, \xi)$ is that the Fredholm determinant $D$ foi the kernel $K_{\xi}(x, \xi)$ shall differ from zero. This kernel, and hence also $D$, depends upon the parameters $\mu$ and $\nu$. We shall show that under the assumption, which we now make, that the given equation is such that

$$
D \not \equiv 0 \text { in } \mu \text { and } \nu,
$$

it is always possible to choose these parameters subject to previous restrictions, so that $D \neq 0$.

We set

$$
\begin{aligned}
K_{\xi}(x, \xi) & =E(x, \xi), \\
K_{\xi}(x, \xi) & =\varepsilon(x, \xi)
\end{aligned}
$$

and denote respectively by $D$ and $D(x, \xi)$ the Fredholm determinant and first minor of the kernel $E(x, \xi)$, and by $\mathcal{D}$ and $\mathcal{D}(x, \xi)$ the corresponding expressions for the kernel $\varepsilon(x, \xi)$. We have*

$$
D=1+\sum_{n=1}^{\infty} d_{n}, \quad D\left(\xi_{1}, \xi_{2}\right)=E\left(\xi_{1}, \xi_{2}\right)+\sum_{n=1}^{\infty} d_{n}\left(\xi_{1}, \xi_{2}\right),
$$

\footnotetext{
* Bocher, An Introduction to Integral Equations, Cambridge University Press, 1909, p. 32.
} 
where, if we omit writing the arguments and indicate them only by their subscripts, thus: $E\left(\xi_{i}, \xi_{j}\right)=E_{i j}$, then

$$
\begin{gathered}
d_{n}=\frac{(-1)^{n}}{n !} \int_{a}^{b} \cdots \int_{a}^{b}\left|\begin{array}{ccc}
E_{11} & \cdots & E_{1 n} \\
\cdot & \cdots & \cdot \\
\cdots & \cdots & \cdot \\
E_{n 1} & \cdots & E_{n n}
\end{array}\right| d \xi_{1} \cdots d \xi_{n}, \\
d_{n}\left(\xi_{1}, \xi_{2}\right)=\frac{(-1)^{n}}{n !} \int_{a}^{b} \cdots \int_{a}^{b}\left|\begin{array}{cccc}
E_{12} & E_{13} & \cdots & E_{1, n+2} \\
E_{32} & E_{33} & \cdots & E_{3, n+2} \\
\cdot & \cdots & \cdots & \cdot \\
\cdot & \cdots & \cdots & \cdot \\
\cdot & \cdots & \cdots & \cdot \\
E_{n+2,2} & \cdots & E_{n+2, n+2}
\end{array}\right| d \xi_{3} \cdots d \xi_{n+2} .
\end{gathered}
$$

We shall omit writing down the analogous formulas for $\mathcal{D}$ and $\mathcal{D}\left(\xi_{1}, \xi_{2}\right)$, since they may be obtained by replacing in those above $d_{n}, d_{n}\left(\xi_{1}, \xi_{2}\right)$ and $E$ respectively by $d_{n}, d_{n}\left(\xi_{1}, \xi_{2}\right)$ and $\varepsilon$.

Consider the term $d_{n}$. By (10) we have

$$
E(x, \xi)=\varepsilon(x, \xi)+\varphi(x) \psi(\xi),
$$

where

$$
\varphi(x) \psi(\xi)=\frac{W(x) V(\xi)}{\Delta} .
$$

Substituting this form and expanding the resulting determinant into a sum of determinants with monomial elements we obtain

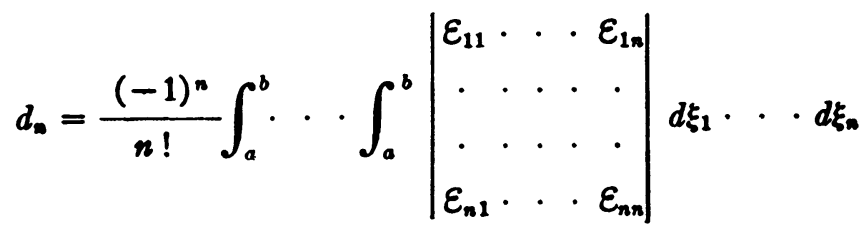

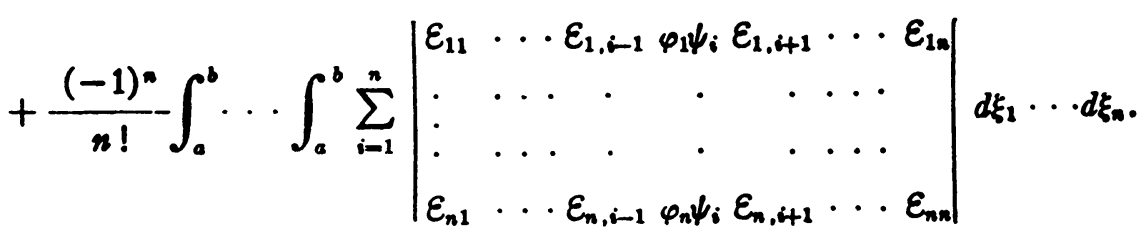


Of the terms on the right the first is simply $d_{n}$. The remaining sum we shall reduce by the following manipulation. In the determinant of the $i$ th term of the sum, let the $i$ th row and column be shifted into first places, and let the arguments be renamed as follows:

$$
\begin{aligned}
& \xi_{k} \text { to become } \xi_{k+1} \text {, for } k=1,2, \cdots,(i-1) \text {; } \\
& \xi_{i} \text { to become } \xi_{1} ;
\end{aligned}
$$

the others to remain unchanged. Since these arguments are all variables of integration this change in their designation amounts merely to a change in the order of integration, and this is immaterial. The terms of the sum have thus been made identical, and hence the entire expression reduces to

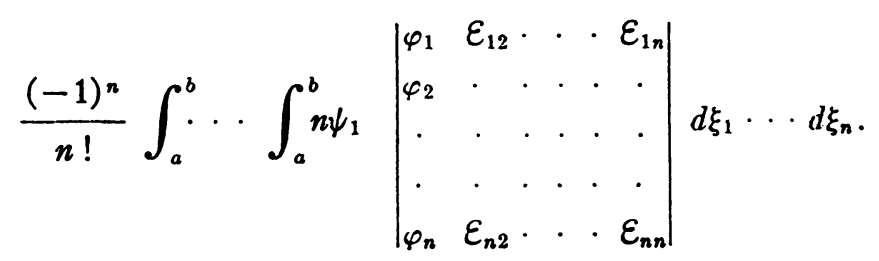

This we expand by the elements of the first column to obtain

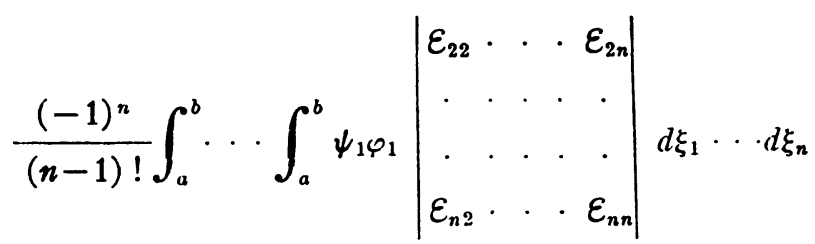

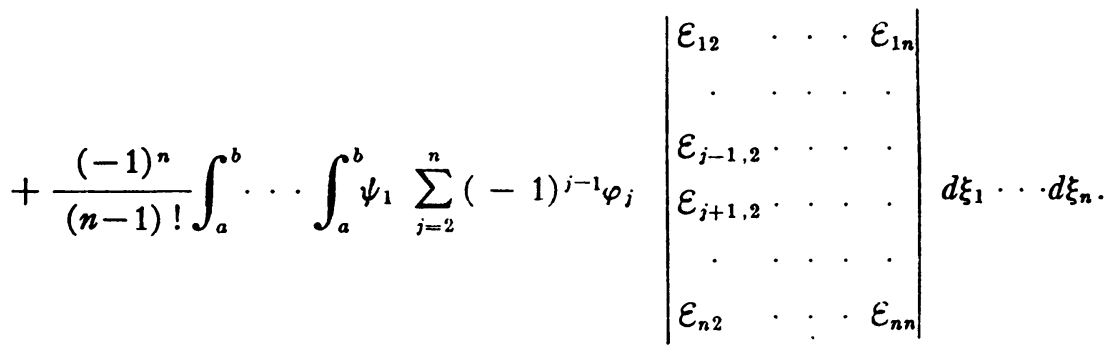

Observing that the first term of this is

$$
-\int_{a}^{b} \varphi\left(\xi_{1}\right) \psi\left(\xi_{1}\right) d_{n-1} d \xi_{1}
$$

we proceed to a further reduction of the remaining sum. Let the $(j-1)$ th column in the respective term (i.e., that in which $j$ is the second subscript) be shifted into first place and let the arguments again be renamed so that $\xi_{k}$ becomes $\xi_{k+1}$, for $k=1,2, \cdots,(j-1)$,

$\xi_{j}$ becomes $\xi_{1}$, 
and the others remain unchanged. This again makes the terms of the sum identical, and the expression takes the form

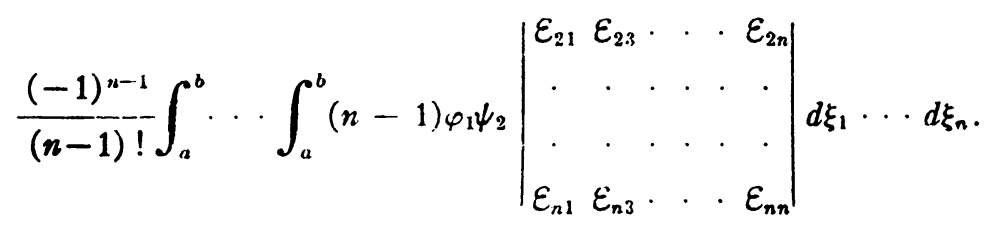

This is precisely

$$
-\int_{a}^{b} \int_{a}^{b} \varphi\left(\xi_{1}\right) \psi\left(\xi_{2}\right) d_{n-2}\left(\xi_{2}, \xi_{1}\right) d \xi_{1} d \xi_{2} .
$$

Collecting our results, then, and summing the $d_{n}$ to obtain $D$, we find that

$$
\left.D=\mathcal{D}-\frac{1}{\Delta} \int_{a}^{b} W\left(\xi_{1}\right)\left\{V^{\prime \prime}\left(\xi_{1}\right) \mathcal{Q}\right)+\int_{a}^{b} I^{\prime \prime}\left(\xi_{2}\right) \mathcal{D}\left(\xi_{2}, \xi_{1}\right) d \xi_{2}\right\} d \xi_{1} .
$$

By use of Fredholm's relation

$$
\varepsilon\left(x, \xi_{1}\right) \mathcal{D}+\int_{a}^{b} \varepsilon\left(x, \xi_{2}\right) \mathcal{D}\left(\xi_{2}, \xi_{1}\right) d \xi_{2}=\mathcal{D}\left(x, \xi_{1}\right),
$$

and the formula

$$
V^{\prime}\left(\xi_{1}\right)=\mu \varepsilon\left(a, \xi_{1}\right)+\nu \varepsilon\left(b, \xi_{1}\right),
$$

which follows from (9), the final form

$$
D=\mathcal{D}-\frac{1}{\Delta} \int_{a}^{b} W\left(\xi_{1}\right)\left\{\mu \mathcal{D}\left(a, \xi_{1}\right)+\nu \mathcal{D}\left(b, \xi_{1}\right)\right\} d \xi_{1}
$$

is obtained. We observe now that in this relation $\Phi$ and $D(x, \xi)$ are free from the parameters $\mu$ and $\nu$, since they depend only on $\varepsilon(x, \xi)$. It follows that $D$ is rational in $\mu$ and $\nu$. The condition $D \neq 0$ in $\mu, \nu$ is found directly to take the explicit form

$$
|\theta(a, b)|+|\theta(a, a+)+\theta(b, b)|+|\theta(b, a)| \neq 0,
$$

where

$$
\theta(x, y)=K(x, y) \mathcal{D}-\int_{a}^{b} \mathcal{D}(x, \xi) K(\xi, y) d \xi,
$$

and $\mathscr{D}$ and $\mathscr{D}(x, \xi)$ are the Fredholm determinant and first minor of the kernel $K_{\xi}(x, \xi)$. Except in the case that this inequality fails, the parameters $\mu$ and $\nu$ can be chosen so that $D \neq 0$. 
The method of manipulation used on $D$ can be employed equally well in the analysis of $D\left(\xi_{1}, \xi_{2}\right)$. It may be shown in this way that $D\left(\xi_{1}, \xi_{2}\right)$ is also rational in $\mu$ and $\nu$.

With $\mu$ and $\nu$ chosen so that $D \neq 0$, the existence of the kernel $F(x, \xi)$ reciprocal to $E(x, \xi)$ is assured. Writing the relation (13) then in "solved" form we have

$$
G(x, \xi)=K(x, \xi)-\int_{a}^{b} F(x, t) K(t, \xi) d t .
$$

9. Characteristics of $F(x, \xi)$. Since the kernel $E(x, \xi)$ is continuous in $R$, it follows that $F(x, \xi)$ is likewise continuous in $R$. The behavior of the partial derivatives of $F(x, \xi)$ may be determined as follows. Differentiating Fredholm's identity

$$
\left.E_{\backslash} \quad \xi\right)+F(x, \xi) \equiv \int_{a}^{b} E(x, t) F(t, \xi) d t \equiv \int_{a}^{b} F(x, t) E(t, \xi) d t,
$$

we obtain

$$
\begin{aligned}
& E_{x}(x, \xi)+F_{x}(x, \xi) \equiv \int_{a}^{b} E_{x}(x, t) F(t, \xi) d t, \\
& E_{\xi}(x, \xi)+F_{\xi}(x, \xi) \equiv \int_{a}^{b} F(x, t) E_{\xi}(t, \xi) d t .
\end{aligned}
$$

Since the right hand members of these relations are obviously continuous it follows that $F_{z}(x, \xi)$ and $F_{\xi}(x, \xi)$ are continuous in $R_{2}$ and $R_{1}^{\prime}$, and hence that

$$
\begin{aligned}
& \left.\left.F_{x}(x, \xi)\right]_{\xi-x}^{\xi-x+} \equiv-E_{x}(x, \xi)\right]_{\xi-x}^{\xi-x+}, \\
& \left.\left.F_{\xi}(x, \xi)\right]_{\xi-x}^{\xi-x+} \equiv-E_{\xi}(x, \xi)\right]_{\xi-x}^{\xi-x+} .
\end{aligned}
$$

In similar manner the higher partial derivatives may be considered. It will be found that $F(x, \xi)$, like $E(x, \xi)$, is possessed in $R_{2}$ and $R_{1}^{\prime}$ of continuous partial derivatives to those of order $(n-1)$.

From the formula

$$
F(x, \xi)=-\frac{D(x, \xi)}{D}
$$

it is seen further that $F(x, \xi)$ is rational in the parameters $\mu$ and $\nu$. From Fredholm's identity above and the relation

$$
\mu E(a, \xi)+\nu E(b, \xi) \equiv 0,
$$


obtained by differentiation from (12), we draw lastly that

$$
\mu F(a, \xi)+\nu F(b, \xi) \equiv 0 .
$$

10. The transformation of the integral equation. Let $u(x)$ represent, now, any solution of the normalized equation (5). Multiplying (14) by $\lambda u(\xi)$ and integrating with respect to $\xi$, we obtain

(18) $\lambda \int_{a}^{b} G(x, \xi) u(\xi) d \xi=\lambda \int_{a}^{b} K(x, \xi) u(\xi) d \xi-\int_{a}^{b} F(x, t) \lambda \int_{a}^{b} K(t, \xi) u(\xi) d \xi \cdot d t$.

In this the integral which occurs in both terms on the right may be evaluated by multiplying (10) by $\lambda u(\xi)$, integrating, and utilizing (5). With the result so obtained and with the abbreviation

$$
L(u)=\mu u(a)+\nu u(b),
$$

the equation (18) reduces to the form

$$
\begin{aligned}
\lambda \int_{a}^{b} G(x, \xi) u(\xi) d \xi= & u(x)-L(u) \frac{W(x)}{\Delta} \\
& -\int_{a}^{b} F(x, t)\left\{u(t)-L(u) \frac{W(t)}{\Delta}\right\} d t .
\end{aligned}
$$

We shall abbreviate this result by setting

$$
\Phi(x)=\frac{1}{\Delta}\left[W(x)-\int_{a}^{b} F(x, t) W(t) d t\right] .
$$

The function $\Phi(x)$ so defined is continuous and has a continuous $(n-1)$ th derivative on $(a, b)$. For subsequent use we observe that

$$
\mu \Phi(a)+\nu \Phi(b)=1 .
$$

Introducing $\Phi(x)$ into (20) we may write that relation

$$
u(x)-\lambda \int_{a}^{b} G(x, \xi) u(\xi) d \xi=L(u) \Phi(x)+\int_{a}^{b} F(x, t) u(t) d t .
$$

By differentiation this yields finally the equation

$$
u^{\prime}(x)-\lambda u(x)=L(u) \varphi(x)+\int_{a}^{b} f(x, t) u(t) d t,
$$

where we have set

$$
\begin{aligned}
\varphi(x) & =\Phi^{\prime}(x), \\
f(x, \xi) & =F_{x}(x, \xi) .
\end{aligned}
$$


Lastly adjoining to the equation thus derived a second equationobviously satisfied by every solution of (5)-we may formulate the result as follows.

THEOREM 2. If the kernel of the normalized integral equation (5) satisfies the conditions (iii),(iv),(v), above, then every solution of the equation is also a solution of the related integro-differential system

$$
\begin{aligned}
& \iota^{\prime}(x)-\lambda u(x)=L(u) \varphi(x)+\int_{a}^{b} f(x, t) u(t) d t \\
& L(u)=\lambda \int_{a}^{b} V(t) u(t) d t .
\end{aligned}
$$

The functions $V(t)$ and $L(u)$ involved here are given respectively by (9) and (19) above. We remark that the function $\varphi(x)$ is continuous together with its derivatives to that of order $(n-2)$ on $(a, b)$, while $f(x, \xi)$ is continuous with its partial derivatives to those of order $(n-2)$ in $R_{2}$ and $R_{1}^{\prime}$.

11. The associated integral equation. Deductions analogous to those above may also be made for the integral equation

$$
v(x)=\lambda \int_{a}^{b} K(\xi, x) v(\xi) d \xi,
$$

associated with equation (5). To accomplish this most easily we shall set $-K(\xi, x)=\bar{K}(x, \xi)$ and write the equation in the form

$$
v(x)=-\lambda \int_{a}^{b} \bar{K}(x, \xi) v(\xi) d \xi,
$$

which is readily found to be its normal form. The deductions already made become applicable to equation $(\overline{5})$, then, if $\lambda$ is replaced by $-\lambda$ and if the functions involved are taken to spring from the kernel $\bar{K}(x, \xi)$ rather than from $K(x, \xi)$. When this is the case we shall indicate it by superscribing the various functional symbols with a bar.

The relation between the two developments is more easily followed if in passing from the treatment of the given equation to that of the associated equation the parameters $\mu$ and $\nu$ are interchanged. We shall suppose this done. Since $\bar{\Delta} \equiv-\Delta$, then, the condition $\bar{\Delta} \neq 0$ has already been met.

It is, however, necessary to impose upon the kernel of the given equation the condition that

$$
\bar{D} \neq 0 \text { in } \nu \text { and } \mu .
$$


The explicit form of this condition as it is obtained from (va) is that

$$
|\bar{\theta}(a, b)|+|\bar{\theta}(a+, a)+\bar{\theta}(b, b)|+|\bar{\theta}(b, a)| \neq 0,
$$

where

$$
\bar{\theta}(x, y)=K(x, y) \overline{\mathcal{D}}-\int_{a}^{b} \overline{\mathcal{D}}(y, \xi) K(x, \xi) d \xi,
$$

and $\overline{\mathcal{D}}$ and $\overline{\mathcal{D}}(x, \xi)$ are the Fredholm determinant and first minor of the kernel $-K_{\xi}(\xi, x)$.

When condition $(\overline{\mathbf{v}})$ is satisfied, every solution of the equation $(\overline{5})$ is found to be a solution of the system

$$
\begin{aligned}
v^{\prime}(x)+\lambda v(x) & =\bar{L}(v) \bar{\varphi}(x)+\int_{a}^{b} \bar{f}(x, t) v(t) d t \\
\bar{L}(v) & =-\lambda \int_{a}^{b} \bar{V}(t) v(t) d t .
\end{aligned}
$$

In this

$$
\bar{L}(v)=\nu v(a)+\mu v(b),
$$

and to obtain the values of $\bar{f}(x, \xi)$ and $\bar{\varphi}(x)$ in terms of familiar symbols we may proceed as follows.

By direct substitution it is found that

$$
\begin{aligned}
\bar{W}(x) & \equiv-V(x), & \bar{V}(\xi) & \equiv-W(\xi), \\
\bar{K}(x, \xi) & \equiv-K(\xi, x), & \bar{E}(x, \xi) & \equiv-K_{\xi}(\xi, x) .
\end{aligned}
$$

Substituting this value of $\bar{E}(x, \xi)$ in Fredholm's identity

$$
\bar{E}(x, \xi)+\bar{F}(x, \xi)=\int_{a}^{b} \bar{E}(x, t) \bar{F}(t, \xi) d t
$$

and differentiating with respect to $x$ we obtain

$$
-K_{\xi x}(\xi, x)+\bar{f}(x, \xi) \equiv-\int_{a}^{b} K_{t x}(t, x) \bar{F}(t, \xi) d t
$$

In this we may replace $K_{\xi x}(\xi, x)$ by its equivalent $E_{\xi}(\xi, x)$. Then further integrating the right hand member of the equation by parts we find that $-E_{\xi}(\xi, x)+\bar{f}(x, \xi)=-E(b, x) \bar{F}(b, \xi)+E(a, x) \bar{F}(a, \xi)+\int_{a}^{b} E(t, x) \bar{f}(t, \xi) d t$. 
Because of (17a) and the formula analogous to (17b), however, the sum of the first two terms on the right vanishes. Hence we have the relation

$$
E_{\xi}(\xi, x)-\bar{f}(x, \xi)=-\int_{a}^{b} \bar{f}(t, \xi) E(t, x) d t .
$$

On the other hand the interchange of the arguments in (15) and the subsequent differentiation of that relation yields

$$
E_{\xi}(\xi, x)+f(\xi, x)=\int_{a}^{b} f(\xi, t) E(t, x) d t .
$$

A comparison of the two results shows that

$$
\bar{f}(x, \xi)=-f(\xi, x) .
$$

With this result and the formulas already noted above we find readily

$$
\bar{\varphi}(x)=\frac{1}{\Delta}\left[V^{\prime}(x)+\int_{a}^{b} V(t) f(t, x) d t\right] .
$$

Lastly we observe that

$$
\left.\vec{f}(x, \xi)]_{\xi-x-}^{\xi-x+}=f(x, \xi)\right]_{\xi-x-}^{\xi-x+} .
$$

Chapter 4. The equivalence of the integro-differential SYSTEM AND THE INTEGRAL EQUATION

12. The transformation of equation (25a). To establish the equivalence of system (25) and equation (5) it remains to show the converse of the theorem of the preceding section, namely that every solution of system (25) is also a solution of equation (5). To do this we shall retrace the steps taken above and so deduce the equation (5) from the system.

Consider first the differential system

$$
\begin{aligned}
& y^{\prime}(x)=\varphi(x), \\
& \mu y(a)+\nu y(b)=1 .
\end{aligned}
$$

Because of (7) it is possessed of a unique continuous solution. By (22) and (24), however, $\Phi(x)$ is such a solution. Hence the system serves to determine $\Phi(x)$ uniquely in terms of $\varphi(x)$. In similar fashion because of (17b) and (24) the system

$$
\begin{aligned}
& \frac{\partial \omega(x, \xi)}{\partial x}=f(x, \xi), \\
& \mu \omega(a, \xi)+\nu \omega(b, \xi)=0
\end{aligned}
$$

serves to determine $F(x, \xi)$ uniquely in terms of $f(x, \xi)$. 
Let $u(x)$ now be any solution of equation (25a), and with the functions $\Phi(x)$ and $F(x, \xi)$ determined construct

$$
\sigma(x)=u(x)-\lambda \int_{a}^{b} G(x, \xi) u(\xi) d \xi-\int_{a}^{b} F(x, \xi) u(\xi) d \xi-L(u) \Phi(x) .
$$

This function is obviously continuous. Moreover, it is a solution of system (6), for we find on the one hand by differentiation the relation

$$
\sigma^{\prime}(x)=u^{\prime}(x)-\lambda u(x)-\int_{a}^{b} f(x, \xi) u(\xi) d \xi-L(u) \varphi(x),
$$

of which the right hand member vanishes by (25a), while it follows on the other hand from property IV of $G(x, \xi)$ and from (17b) and (22) that

$$
\mu \sigma(a)+\nu \sigma(b)=0 .
$$

The system (6) is incompatible, however. Hence $\sigma(x) \equiv 0$, that is, $u(x)$ satisfies equation (23).

The function $F(x, \xi)$ was originally derived in Chapter 3 as a reciprocal kernel. Hence it is itself possessed of a reciprocal, which is, moreover, precisely $E(x, \xi)$ of the preceding chapter. The integral equations

$$
\begin{gathered}
\theta(x)=\Phi(x)+\int_{a}^{b} F(x, t) \theta(t) d t, \\
\omega(x, \xi)=G(x, \xi)+\int_{a}^{b} F(x, t) \omega(t, \xi) d t
\end{gathered}
$$

are, therefore, uniquely solvable, and since by (21) and (14) we have as solutions respectively $W(x) / \Delta$ and $K(x, \xi)$, these functions are uniquely determinable. We have at our disposal, therefore, the relations (14) and (21) satisfied by them, while the substitution of (21) in (23) yields further the relation $(20)$.

Let (14) be written, now, in "solved" form, thus:

$$
K(x, \xi)=G(x, \xi)-\int_{a}^{b} E(x, t) G(t, \xi) d t .
$$

From this we obtain upon multiplying it by $\lambda u(\xi)$ and integrating it with respect to $\xi$

$$
\lambda \int_{a}^{b} K(x, \xi) u(\xi) d \xi=\lambda \int_{a}^{b} G(x, \xi) u(\xi) d \xi-\int_{a}^{b} E(x, t) \cdot \lambda \int_{a}^{b} G(t, \xi) u(\xi) d \xi d t .
$$


We shall eliminate from this the quantity

$$
\lambda \int_{a}^{b} G(x, \xi) u(\xi) d \xi,
$$

by substituting for it its value as given by (20). In this way we find, upon collecting terms, that

$$
\begin{gathered}
\lambda \int_{a}^{b} K(x, \xi) u(\xi) d \xi=u(x)-L(u) \frac{W(x)}{\Delta} \\
-\int_{a}^{b}\left\{F(x, \xi)+E(x, \xi)-\int_{a}^{b} E(x, t) F(t, \xi) d t\right\}\left\{u(\xi)-L(u) \frac{W(\xi)}{\Delta}\right\} d \xi .
\end{gathered}
$$

This reduces, however, for by Fredholm's identity (15) the first factor in the integrand on the right vanishes. It follows that every solution of (25a) satisfies also the equation

$$
u(x)=\lambda \int_{a}^{b} K(x, \xi) u(\xi) d \xi+L(u) \frac{W(x)}{\Delta} .
$$

13. Application of equation (25b). If the solution $u(x)$ of equation (25a) satisfies also (25b), the value of $L(u)$ may be eliminated between $(25 \mathrm{~b})$ and (29) above. The result obtained is that

$$
u(x)=\lambda \int_{a}^{b}\left\{K(x, \xi)+\frac{W(x) V(\xi)}{\Delta}\right\} u(\xi) d \xi,
$$

and since the bracket in the integrand is, by $(10), K(x, \xi)$, this is precisely equation (5). This result we formulate as follows.

THEOREM 3. Under the hypotheses of Theorem 2 every solution of the integrodifferential system (25) is also a solution of the related normalized integral equation (5).

The proof of the equivalence of system (25) and equation (5) is thus completed.

14. Generalization. In the deductions of this chapter we have been concerned hitherto with the particular system (25) derived in Chapter 3 from equation (5), and because of this we were able to identify as previously known functions the solutions of the various defining differential systems and integral equations. In any case, however, we see from equation (30) and the fact that $W(x) / \Delta$ and $K(x, \xi)$ there involved are solutions of the respective 
equations (28), that the kernel of equation (30) is itself given as the solution of the integral equation

$$
K(x, \xi)=[G(x, \xi)+\Phi(x) V(\xi)]+\int_{a}^{b} F(x, l) \kappa(t, \xi) d t
$$

It is clear from this that the method may be applied to the transformation of the general system of type (25), provided that the function $F(x, \xi)$ defined as above is possessed of a reciprocal. We may state therefore the following theorem.

Theorem 4. If an integro-differential system of type (25) satisfies the condition that the solution of its related differential system (27) is possessed of a reciprocal, then every solution of the integro-differential system solves also a related linear integral equation of the second kind.

15. The associated system. Clearly all the methods employed above are applicable to the system $(\overline{2} \overline{5})$ as well as to $(25)$. Hence we may conclude that system $(\overline{2} \overline{5})$ is equivalent to the equation $(\overline{5})$.

Chapter 5. The existence of a solution of the integro-differENTIAL EQUATION FOR LARGE VALUES OF THE PARAMETER

16. Matters of notation. For the sake of simplicity in the formulations and deductions of this and subsequent chapters we shall make here certain conventions of notation. To begin with, the relation $|\lambda|>N$ shall be interpreted as an abbreviation of the statement " $|\lambda|$ sufficiently large." It is to be understood, therefore, that $N$ does not necessarily mean the same constant in any one case as it does in any other. Further we reserve $B(x, \xi, \lambda)$ as a generic symbol for functions which for $(x, \xi)$ in the region $R$ and $|\lambda|>N$ are possessed of the following properties:

(a) $B(x, \xi, \lambda)$ is uniformly bounded, i.e.

$$
|B(x, \xi, \lambda)|<B \text { (a consiant); }
$$

(b) $B(x, \xi, \lambda)$ is integrable in $\xi$ uniformly in $x$ and $\lambda$, that is, if the interval $(a, b)$ is subdivided by the points $\xi_{0}=a<\xi_{1}<\xi_{2}<\cdots<\xi_{n}=b$ in any manner so that $\left|\xi_{i}-\xi_{i-1}\right|$ approaches zero as $n \rightarrow \infty$, and if $U_{i}(x, \lambda)$ and $L_{i}(x, \lambda)$ are respectively the upper and lower bounds of $B(x, \xi, \lambda)$ on the sub-interval $\left(\xi_{i-1}, \xi_{i}\right)$, then when any constant $\epsilon>0$ is prescribed there exists a numbe $\boldsymbol{n}_{1}$ such that

$$
\left|\sum_{i=1}^{n}\left[U_{i}(x, \lambda)-L_{i}(x, \lambda)\right]\left(\xi_{i}-\xi_{i-1}\right)\right|<\epsilon
$$

for $n \geqq n_{1}$. 
Lastly $\sigma$ and $i \tau$ will be used to designate the real and pure imaginary parts of $\lambda$, thus: $\lambda=\sigma+i \tau$, and a constant designated $M$ shall be understood to be positive or zero.

17. Lemmas. For use in the subsequent deductions we begin by establishing the following lemmas.

LEMma 1. If $a \leqq z \leqq b$ and

then

$$
I(x, z, \lambda)=\int_{a}^{z} e^{\lambda(z-\xi)} B(x, \xi, \lambda) d \xi,
$$

$$
\lim _{\substack{\mid \lambda \rightarrow \infty \\ \sigma \leqq M}} I=0
$$

uniformly in $x$ and $z$ and the argument of $\lambda$.

To establish this let the interval $(a, b)$ be subdivided in the manner described under (b) above, the point $z$ being taken as one of the points of subdivision, i.e. $\xi_{k}=z$. Then if $\bar{\xi}_{i}$ is any point of the interval $\left(\xi_{i-1}, \xi_{i}\right)$ we have for $\xi$ on this interval

$$
\left|B(x, \xi, \lambda)-B\left(x, \bar{\xi}_{i}, \lambda\right)\right|<U_{i}(x, \lambda)-L_{i}(x, \lambda) .
$$

Let $I(x, z, \lambda)$ be written now in the form

$$
\begin{aligned}
I(x, z, \lambda)=\sum_{i=1}^{k} & \left\{B\left(x, \bar{\xi}_{i}, \lambda\right) \int_{\xi_{i-1}}^{\xi_{i}} e^{\lambda(z-\xi)} d \xi\right. \\
& \left.+\int_{\xi_{i-1}}^{\xi_{i}} e^{\lambda(z-\xi)}\left[B(x, \xi, \lambda)-B\left(x, \bar{\xi}_{i}, \lambda\right)\right] d \xi\right\} .
\end{aligned}
$$

Then since

$$
\left|e^{\lambda(z-a)}\right| \leqq e^{M(b-a)} \text { for } \sigma \leqq M, \quad a \leqq \xi \leqq z \leqq b,
$$

we have

$$
\begin{aligned}
&|I(x, z, \lambda)| \leqq \sum_{i=1}^{k}\left\{B \cdot\left|\frac{e^{\lambda\left(z-\xi_{i-1}\right.}-e^{\lambda\left(z-\xi_{i}\right)}}{\lambda}\right|\right. \\
&\left.+e^{M(b-a)}\left[U_{i}(x, \lambda)-L_{i}(x, \lambda)\right] \int_{\xi_{i-1}}^{\xi_{i}} d \xi\right\} \\
& \leqq \frac{2 k B e^{M(b-a)}}{|\lambda|}+e^{M(b-a)} \sum_{i=1}^{k}\left[U_{i}(x, \lambda)\right. \\
&\left.-L_{i}(x, \lambda)\right]\left(\xi_{i}-\xi_{i-1}\right) .
\end{aligned}
$$


Now let $\epsilon>0$ be arbitrarily chosen. Then since $k \leqq n$ the final sum will, by hypothesis (b), be less than $\epsilon$ for $n \geqq n_{1}$, and we have

$$
|I(x, z, \lambda)| \leqq e^{M(b-a)}\left[\frac{2 n_{1} B}{|\lambda|}+\epsilon\right] .
$$

Since $n_{1}$ is fixed when $\epsilon$ is chosen, we may by making $|\lambda|$ sufficiently large reduce the right hand member of the inequality to less than $2 e^{\boldsymbol{M ( b - a )}} \epsilon$. This proves the lemma.

\section{LEMMa 2. If}

$$
I_{2}(x, z, \lambda)=\int_{b}^{z} e^{\lambda(z-\xi)} B(x, \xi, \lambda) d \xi
$$

then

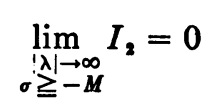

uniformly in $x$ and $z$ and the argument of $\lambda$.

The proof of this lemma is in every way similar to that of Lemma 1.

18. Transformation of the integro-differential equation. We consider now the integro-differential equation

$$
u^{\prime}(x)-\lambda u(x)=L(u) \varphi(x)+\int_{a}^{b} f(x, \xi) u(\xi) d \xi+E(x, \lambda) .
$$

The symbol $E(x, \lambda)$ is to be considered generical, designating a uniformly bounded function which is analytic in $\lambda$ for $|\lambda|>N$. The equation (32) reduces to equation $(25 \mathrm{a})$ in the special case $E(x, \lambda) \equiv 0$. For subsequent applications it is essential to consider the more general case.

If we consider equation (32) for the moment as a non-homogeneous equation of the type

$$
u^{\prime}+p u=q,
$$

it follows from the theory of differential equations that it may be written in the form

$$
u(x)=c e^{\lambda x}+\int_{*}^{x} e^{\lambda(x-t)}\left\{L(u) \varphi(t)+\int_{a}^{b} f(t, \xi) u(\xi) d \xi+E(t, \lambda)\right\} d t,
$$

the limit $*$ being any constant on the interval $(a, b)$, and the coefficient $c$ being constant with respect to $x$ but otherwise arbitrary.

For purposes of orientation we shall proceed to deduce the consequences of the assumption that for such choices of the constants as will be made, equation (32a) is possessed of a solution. This deduction will be made 
separately for the cases in which $\lambda$ is confined to a left hand half-plane $\sigma \leqq M$, and in which $\lambda$ is confined to a right hand half-plane $\sigma \geqq-M$.

19. Heuristic deductions when $\sigma \leqq M$. Let the arbitrary elements in (32a) be chosen in this case as follows:

$$
*=a, \quad c=c_{1} e^{-\lambda a},
$$

where $c_{1}$ is any absolute constant. The equation becomes, then,

(32b) $u(x)=c_{1} e^{\lambda(x-a)}+\int_{a}^{x} e^{\lambda(x-t)}\left\{L(u) \varphi(t)+\int_{a}^{b} f(t, \xi) u(\xi) d \xi+E(t, \lambda)\right\} d t$.

Substituting the right hand member of this for $u(x)$ in the expression $L(u)$, we obtain, upon solving for $L(u)$,

$$
L(u)=C^{-1}\left\{L\left(c_{1} e^{\lambda(x-a)}\right)+\nu \int_{a}^{b} e^{\lambda(b-t)}\left\{E(t, \lambda)+\int_{a}^{b} f(t, \xi) u(\xi) d \xi\right\} d t\right\}
$$

where

$$
C=\left[1-\nu \int_{a}^{b} e^{\lambda(t-t)} \varphi(t) d t\right] .
$$

This solution is possible if $|\lambda|>N$. For, since $\varphi(\xi)$ is a function of the type denoted by $B(x, \xi, \lambda)$ the integral in the expression for $C$ decreases to zero as $|\lambda|$ increases, by Lemma 1 . Hence $C \neq 0$ for $|\lambda|>N$.

With the value of $L(u)$ thus obtained equation (32b) takes the form

$$
u(x)=\theta(x, \lambda)+\int_{a}^{b} \Omega(x, \xi, \lambda) u(\xi) d \xi,
$$

where

$$
\begin{aligned}
\theta(x, \lambda)= & c_{1} e^{\lambda(x-a)}+\int_{a}^{x} e^{\lambda(x-t)} E(t, \lambda) d t \\
& +C^{-1}\left\{L\left(c_{1} e^{\lambda(x-a)}\right)+\nu \int_{a}^{b} e^{\lambda(b-1)} E(t, \lambda) d t\right\} \cdot \int_{a}^{x} e^{\lambda(x-t)} \varphi(t) d t,
\end{aligned}
$$

and

$$
\Omega(x, \xi, \lambda)=\int_{a}^{x} e^{\lambda(x-l)} f(t, \xi) d t+C^{-1} \nu \int_{a}^{b} e^{\lambda(b-t)} f(t, \xi) d t \cdot \int_{a}^{x} e^{\lambda(x-t)} \varphi(t) d t .
$$

The assumed solution of equation (32b) must, therefore, satisfy also equation (32c).

20. The existence of a solution of equation (32) for $\sigma \leqq M$. Returning now to equation (32a) we may construct, by the respective formulas above, 
the constant $C$, the functions $\theta(x, \lambda)$ and $\Omega(x, \xi, \lambda)$, the equation (32c), and the series

$$
\begin{aligned}
\theta(x, \lambda) & +\int_{a}^{b} \Omega(x, \xi, \lambda) \theta(\xi, \lambda) d \xi \\
& +\int_{a}^{b} \Omega(x, \xi, \lambda) \int_{a}^{b} \Omega\left(\xi, \xi_{1}, \lambda\right) \theta\left(\xi_{1}, \lambda\right) d \xi_{1} d \xi+\cdots
\end{aligned}
$$

In the formulas for $\theta(x, \lambda)$ and $\Omega(x, \xi, \lambda)$ the integrals involving $E(t, \lambda)$ are uniformly bounded. The remaining integrals may be made arbitrarily small by taking $|\lambda|>N$, since $f(x, \xi)$, like $\varphi(\xi)$, is a function of the type $B(x, \xi, \lambda)$. Lastly $e^{\lambda(x-a)}$ is bounded, since $\sigma \leqq M$. Hence for $|\lambda|>N$

$$
\begin{gathered}
|\theta(x, \lambda)|<A \quad(\text { a constant }), \\
|\Omega(x, \xi, \lambda)|<\frac{1}{2(b-a)},
\end{gathered}
$$

and the terms of the series (34) are in absolute value less than the corresponding terms of the series

$$
A+\frac{A}{2}+\frac{A}{2^{2}}+\cdots
$$

The series (34) converges, therefore, uniformly to a function numerically less than $2 A$. Since the terms of (34) are continuous in $x$ and analytic in $\lambda$ it follows that (34) converges to a function $u(x, \lambda)$ which is uniformly bounded, continuous in $x$ and analytic in $\lambda$. On the other hand by the classical theory of integral equations* this function $u(x, \lambda)$ is a solution of (32c).

We observe that if $E(x, \lambda) \equiv 0$, then the choice $c_{1}=0$ leads to $\theta(x, \lambda) \equiv 0$. The solution found is in this case $u(x, \lambda) \equiv 0$. We shall suppose, therefore, in proceeding, that $c_{1}$ has been so chosen that $\left|c_{1}\right|+|E(x, \lambda)| \not \equiv 0$.

Let the right hand member of (32c) be substituted now for $u(x)$ in the expression $L(u)$. The result is found to be precisely (33) above. The right hand member of (33) is contained, however, in the right hand member of (32c). The elimination of it between the two equations yields equation (32b), which is, therefore, satisfied by the function $u(x, \lambda)$ found. Lastly differentiating (32b) we obtain equation (32), which completes the proof that for $\sigma \leqq M,|\lambda|>N$, equation (32) is possessed of a solution which is uniformly bounded, continuous in $x$, and analytic in $\lambda$.

*Bócher, loc. cit., p. 15. 
21. The case $\sigma \geqq-M$. When $\lambda$ is to be confined to a right hand half plane $\sigma \geqq-M$ the arbitrary elements in (32a) may be chosen as follows:

$$
*=b, \quad c=c_{2} e^{-\lambda b} \text {, }
$$

where $c_{2}$ is any absolute constant. Reasoning precisely analogous to that followed in $\S 19$ and $\S 20$ serves, then, to establish the fact that for $\sigma \geqq-M$ and $|\lambda|>N$ equation (32) is also possessed of a solution with the characteristics noted above.

22. The associated equation. The discussion of the equation obtained from $(\overline{25 a})$ by adding on the right a function $E(x, \lambda)$ may be carried through precisely like the discussion just concluded. In this way it is found that for $\lambda$ confined to any half-plane $\sigma \leqq M$ or $\sigma \geqq-M$, and $|\lambda|>N$, such an equation also is possessed of a uniformly bounded solution which is analytic in $\lambda$ and continuous in $x$.

Summarizing the results of this chapter we have obtained the following theorem.

THEOREM 5. If the complex parameter $\lambda$ is restricted to a region bounded by a line parallel to the axis of imaginaries and exterior to a circle sufficiently large and with center at $\lambda=0$, then the integro-differential equation (32) admits of a solution which is uniformly bounded, continuous in $x$, and analytic in $\lambda$.

Chapter 6. The formal SOlution of the INTEgro-differential

$$
\text { EQUATION }
$$

23. A lemma. In order to preclude interruption of the deductions about to be made we begin by establishing the following lemma. The symbol $\boldsymbol{H}(x, \xi, \lambda)$ will be used to designate a function which is merely bounded uniformly for $|\lambda|>N$, and is integrable in $x$ and $\xi$.

LEMMA 3. If $z_{1}$ and $z_{2}$ are any points of the interval $(a, b)$, and

$$
I\left(z_{1}, z_{2}, \xi, \lambda\right)=\int_{z_{1}}^{z_{2}} e^{\lambda t} H(\xi, t, \lambda) d t,
$$

then the functional form of this integral is given by

$$
I\left(z_{1}, z_{2}, \xi, \lambda\right)=e^{\lambda z_{2}} H\left(z_{2}, \xi, \lambda\right)+e^{\lambda z_{1}} H\left(z_{1}, \xi, \lambda\right) .
$$

This lemma follows almost immediately from the preceding ones. Thus the integral may be written in the form

$$
I\left(z_{1}, z_{2}, \xi, \lambda\right)=e^{\lambda z_{2}} \int_{*}^{z_{2}} e^{-\lambda\left(z_{2}-t\right)} H(\xi, t, \lambda) d t-e^{\lambda z_{1}} \int_{*}^{z_{1}} e^{-\lambda\left(z_{2}-t\right)} H(\xi, t, \lambda) d t,
$$


where * denotes any constant on the interval $(a, b)$. If $-\sigma \leqq M$ we may choose $*=a$. Then the factors of the integrands on the right are all uniformly bounded. The same is therefore obviously true of the integrals themselves. On the other hand if $-\sigma \geqq-M$ we may choose $*=b$, with the result that the integrals are again uniformly bounded. This proves the lemma.

24. Heuristic investigation of the functional form of a solution of (25a). The existence of a solution of (25a) has been shown. We shall suppose now for purposes of orientation that such a solution may be obtained by the method of successive approximations, and in particular by the approximating scheme defined by the formula

$$
u_{i}^{\prime}(x)-\lambda u_{i}(x)=L\left(u_{i-1}\right) \varphi(x)+\int_{a}^{b} f(x, \xi) u_{i-1}(\xi) d \xi .
$$

This formula is in the form of a differential equation for $u_{i}(x)$ when $u_{i-1}(x)$ is known.

With the initial approximating function $u_{0} \equiv 0$ we have as a possible choice $u_{1}=e^{\lambda x}$. Substituting this in the right hand member of (35) and applying Lemma 3 we find that $u_{2}(x)$ is given by an equation of the type

$$
u_{2}^{\prime}(x)-\lambda u_{2}(x)=e^{\lambda b} H(x, \lambda)+e^{\lambda a} H(x, \lambda) .
$$

The formula

$$
u_{2}(x)=e^{\lambda x}+\int_{*}^{x} e^{\lambda(x-t)}\left\{e^{\lambda b} H(t, \lambda)+e^{\lambda a} H(t, \lambda)\right\} d t
$$

yields a solution when the $*$ is chosen arbitrarily for each term of the integrand. We obtain a particular solution $u_{2}(x)$, then, by choosing $*=b$ for the terms of which $e^{\lambda b}$ is a factor, and $*=a$ for the terms which contain $e^{\lambda}$. By Lemma (3), then

$$
u_{2}(x)=e^{\lambda b} H(x, \lambda)+e^{\lambda x} H(x, \lambda)+e^{\lambda a} H(x, \lambda) .
$$

Upon the substitution of this as $u_{i-1}(x)$ in (35) and the application of Lemma 3 we find that $u_{3}(x)$ satisfies an equation of precisely the type (35a). Hence there exists a $u_{3}(x)$ of the same functional character as $u_{2}(x)$ given by (36). Obviously the same argument may be applied in the course of each successive substitution, and since we may consequently choose $u_{n}(x)$ for every $n$ of the form of $u_{2}(x)$ above, the convergence of the procedure would lead to a solution of equation (35a) of the form (36). Guided by this result we proceed to substitute the form (36) into the equation with the purpose of determining as far as possible the functions $H(x, \lambda)$. 
25. The formal determination of coefficients. The undetermined functions $H(x, \lambda)$ are bounded for $|\lambda|>N$. We shall proceed on the hypothesis that they are expansible in power series in $1 / \lambda$, and shall write the assumed form of the solution of (25a)

$$
u(x)=e^{\lambda x}[\gamma(x)]+e^{\lambda b}[\beta(x)]+e^{\lambda a}[\alpha(x)] .
$$

In the notation used here

$$
[\omega(x)]=\sum_{i=0}^{\infty} \frac{\omega_{i}(x)}{\lambda} .
$$

The result of the substitution of form (37) in the equation (25a) involves the integral

$$
I=\int_{a}^{b} e^{\lambda \xi} f(x, \xi)[\gamma(\xi)] d \xi .
$$

We shall consider this in two parts, namely

and

$$
I_{1}=\int_{a}^{b} e^{\lambda \xi} f(x, \xi) \sum_{k=0}^{n-2} \frac{\gamma_{k}(\xi)}{\lambda^{k}} d \xi,
$$

$$
I_{2}=\int_{a}^{b} e^{\lambda \xi} f(x, \xi) \sum_{k=n-1}^{\infty} \frac{\gamma_{k}(\xi)}{\lambda^{k}} d \xi
$$

Integrating the $k$ th term of $I_{1}$ by parts $(n-2-k)$ times, we obtain the formula

$$
\begin{gathered}
I_{1}=\sum_{k=0}^{n-3} \sum_{i=0}^{n-3-k}(-1)^{i+1} \frac{e^{\lambda b} f \gamma_{k}^{i}(x, b)-e^{\lambda x} J f \gamma_{k}^{i}-e^{\lambda a} f \gamma_{k}^{i}(x, a)}{\lambda^{k+i+1}} \\
+\frac{(-1)^{n-2}}{\lambda^{n-2}} \int_{a}^{b} e^{\lambda \xi} \sum_{k=0}^{n-2}(-1)^{k} f \gamma_{k^{n-2-k}}(x, \xi) d \xi .
\end{gathered}
$$

The notation has been abbreviated here so that

$$
\begin{aligned}
f(x, \xi) \gamma_{k}(\xi) & =f \gamma_{k}(x, \xi), \\
\left.f \gamma_{k}(x, \xi)\right]_{\xi=x}^{\xi=x+} & =J f \gamma_{k}, \\
\frac{\partial^{i} f \gamma_{k}(x, \xi)}{\partial \xi^{i}} & =f \gamma_{k}^{i}(x, \xi) .
\end{aligned}
$$


The substitution of (37) in the equation yields therefore

$$
\begin{aligned}
u^{\prime}(x) & -\lambda u(x)-L(u) \varphi(x)-\int_{a}^{b} f(x, \xi) u(\xi) d \xi \\
= & e^{\lambda x}\left\{\left[\gamma^{\prime}(x)\right]+\sum_{k=0}^{n-3} \sum_{i=0}^{n-3-k}(-1)^{i} \frac{J f_{k}^{i}}{\lambda^{k+i+1}}\right\}+e^{\lambda b}\{\} \\
& +e^{\lambda a}\{\}+\frac{(-1)^{n-2}}{\lambda^{n-2}} \int_{a}^{b} e^{\lambda \xi} \sum_{k=0}^{n-2}(-1)^{k} f \gamma_{k^{n-2-k}}(x, \xi) d \xi \\
& +\int_{a}^{b} e^{\lambda \xi} f(x, \xi) \sum_{k=n-1}^{\infty} \frac{\gamma_{k}(\xi)}{\lambda^{k}} d \xi=0,
\end{aligned}
$$

where the coefficients of $e^{\lambda b}$ and $e^{\lambda a}$ are of the same general character as that of $e^{\lambda x}$.

Equating now to zero the coefficient of $e^{\lambda x} / \lambda^{l}$ for $l=0,1,2, \cdots,(n-2)$ respectively we find that $\gamma_{0}{ }^{\prime} \equiv 0$, whence we may choose $\gamma_{0}=1$, and that the functions $\gamma_{1}(x) \gamma_{2}(x), \cdots, \gamma_{n-2}(x)$ can be successively determined each by means of a quadrature. Continuing in similar fashion and equating to zero the coefficients of $e^{\lambda b} / \lambda^{l}$ and $e^{\lambda a} / \lambda^{l}$ for $l=-1,0,1, \cdots,(n-3)$, respectively, we find likewise that

$$
\begin{aligned}
\beta_{0}= & 0, \\
\beta_{1}(x)=-\nu \varphi(x), & \text { etc. } \\
\alpha_{0}= & 0, \\
\alpha_{1}(x)= & -\mu \varphi(x), \\
& \text { etc. },
\end{aligned}
$$

and that $\beta_{2}, \cdots, \beta_{n-2}, \alpha_{2}, \cdots, \alpha_{n-2}$ may also be successively evaluated. Hence we may construct the function $\bar{u}(x)$ given by the formula

$$
\begin{aligned}
\tilde{u}(x)= & e^{\lambda x}\left\{1+\frac{\gamma_{1}(x)}{\lambda}+\cdots+\frac{\gamma_{n-2}(x)}{\lambda^{n-2}}\right\} \\
& +e^{\lambda b}\left\{\frac{\beta_{1}(x)}{\lambda}+\cdots+\frac{\beta_{n-2}(x)}{\lambda^{n-2}}\right\} \\
& +e^{\lambda a}\left\{\frac{\alpha_{1}(x)}{\lambda}+\cdots+\frac{\alpha_{n-2}(x)}{\lambda^{n-2}}\right\} .
\end{aligned}
$$

26. The equation satisfied by $\bar{u}(x)$. The deductions above have been purely formal. A consideration of formulas involved shows, however, that 
since $\varphi(x)$ is possessed of derivatives of order $(n-2)$, while $f(x, \xi)$ is possessed of partial derivatives of the same order, $\gamma_{i}(x)$ may be differentiated $(n-i)$ times, while $\beta_{i}(x)$ and $\alpha_{i}(x)$ are differentiable $(n-1-i)$ times. The function $\bar{u}(x)$ determined is, therefore, differentiable and can be substituted into equation (25a).

The result of this substitution may be read directly from (38) above if the coefficients with subscripts greater than $(n-2)$ are taken now to be zero, and the symbols $[\gamma(x)]$ etc. are interpreted as representing the respective polynomials instead of the formal infinite series. The coefficients have been explicitly determined so that the terms in $e^{\lambda x} / \lambda^{i}, e^{\lambda b} / \lambda^{i-1}$, and $e^{\lambda a} / \lambda^{i-1}$ vanish for $i=0,1, \cdots,(n-2)$. Since the integral $I_{2}$ of (38) does not now appear, there remain merely a term of the form $B(x) e^{\lambda b} / \lambda^{n-2}$, an analogous term in $e^{\lambda a} / \lambda^{n-2}$, and the integral

$$
\frac{(-1)^{n-2}}{\lambda^{n-2}} \int_{a}^{b} e^{\lambda \xi} \sum_{k=0}^{n-2}(-1)^{k} f \gamma k^{n-2-k}(x, \xi) d \xi .
$$

Writing this integral in the form used in the proof of Lemma 3 we find readily that the substitution yields the result

$$
\begin{gathered}
\bar{u}^{\prime}(x)-\lambda \bar{u}(x)-L(\bar{u}) \varphi(x)-\int_{a}^{b} f(x, \xi) \bar{u}(\xi) d \xi \\
=\frac{1}{\lambda^{n-2}}\left\{e^{\lambda b} B(x, \lambda)+e^{\lambda a} B(x, \lambda)\right\},
\end{gathered}
$$

where, moreover, the functions $B(x, \lambda)$ are analytic in $\lambda$. We shall call the function $\bar{u}(x)$ a formal solution to the order $(n-2)$ of equation (25a), since for such values of $\lambda$ for which the exponential factors in (41) are bounded the equations (41) and (25a) agree to terms of degree $(n-2)$ in $(1 / \lambda)$.

27. The associated equation. Equation $(\overline{25 a})$ differs from equation (25a) in that each function involved is replaced by the corresponding function superscribed with a bar, and in that $\lambda$ is replaced by $-\lambda$. With these alterations formula (40) above yields the formal solution of $(\overline{25 a})$. Because of relation (26b), therefore, this formal solution is

$$
\begin{aligned}
\bar{v}(x)= & e^{-\lambda x}\left\{1-\frac{\gamma_{1}(x)}{\lambda}+\cdots+(-1)^{n-2} \frac{\bar{\gamma}_{n-2}(x)}{\lambda^{n-2}}\right\} \\
& +e^{-\lambda b}\left\{-\frac{\bar{\beta}_{1}(x)}{\lambda}+\cdots+(-1)^{n-2} \frac{\bar{\beta}_{n-2}(x)}{\lambda^{n-2}}\right\} \\
& +e^{-\lambda a}\left\{-\frac{\bar{\alpha}_{1}(x)}{\lambda}+\cdots+(-1)^{n-2} \frac{\bar{\alpha}_{n-2}(x)}{\lambda^{n-2}}\right\},
\end{aligned}
$$


the function $\gamma_{1}(x)$ occurring here being that which occurs also in formula (40).

Chapter 7. The solution of the INTEgro-differential equation

28. Relation of the formal solution to a true solution for $\sigma \leqq M$. By the conclusions of $\S 20$ equation (25a) admits, for $\sigma \leqq M,|\lambda|>N$, of a solution $\boldsymbol{u}(x)$ which satisfies the relation (32b) with $c_{1}$ any non-vanishing constant, and $E \equiv 0$. Such a solution will not vanish for $x=a$, and hence upon being multiplied by a suitable function of $\lambda$ alone, will satisfy the condition

$$
\boldsymbol{u}(a)=\bar{u}(a),
$$

where $\bar{u}(x)$ is the formal solution (40). This multiplication by a function independent of $x$ is permissible since the equation (25a) is homogeneous. Inasmuch as the solution of (32b) originally chosen is analytic in $\lambda$ for $|\lambda|>N$, while the same is true of the formal solution $\bar{u}(x)$, the analyticity of the final form $u(x)$ is assured.

Consider now the function

$$
w(x)=\lambda^{n-2} e^{-\lambda a}\{u(x)-\bar{u}(x)\} .
$$

The bracket on the right obviously satisfies equation (41). Hence $w(x)$ is a solution of an equation

$$
w^{\prime}(x)-\lambda w(x)=L(w) \varphi(x)+\int_{a}^{b} f(x, \xi) w(\xi) d \xi+\left\{e^{\lambda(b-a)} B(x, \lambda)+B(x, \lambda)\right\},
$$

the functions $B(x, \lambda)$ being analytic in $\lambda$. This equation is of the form (32) since for $\sigma \leqq M$ the bracket on the right is of the type $E(x, \lambda)$, and hence we may apply to it the deductions of $\S 19$ and $\S 20$. Since $w(a)=0$ by (42), we find from the form (32b) that we have before us a case in which the coefficient $c_{1}$ of $\S 19$ is zero. The function $\theta(x, \lambda)$ reduces, therefore, in this case to

$$
\begin{aligned}
\theta(x, \lambda)= & \int_{a}^{x} e^{\lambda(x-t)}\left\{e^{\lambda(b-a)} B(t, \lambda)+B(t, \lambda)\right\} d t \\
& +C^{-1} \nu \int_{a}^{b} e^{\lambda(b-t)}\left\{e^{\lambda(b-a)} B(t, \lambda)+B(t, \lambda)\right\} d t \cdot \int_{a}^{x} e^{\lambda(x-t)} \varphi(t) d t,
\end{aligned}
$$

and hence is, by Lemma 1 , of the form $\epsilon(x, \lambda)$, where this symbol is used to denote a function which approaches zero uniformly in $x$ as $|\lambda| \rightarrow \infty$. In consequence of this, as may be drawn from $\S 20, w(x)$ is itself of the form $\epsilon(x, \lambda)$. 
Solving (42), then, for $u(x)$ we obtain the result that there exists for $\sigma \leqq M,|\lambda|>N$, a solution of the form

$$
u(x)=\bar{u}(x)+\frac{\epsilon(x, \lambda) e^{\lambda \varepsilon}}{\lambda^{n-2}} .
$$

This solution is moreover continuous in $x$ and analytic in $\lambda$.

29. The solution for $\sigma \geqq-M$. The deductions of $\S 21$ establish the existence of a solution of equation (25a) which for $\sigma \geqq-M,|\lambda|>N$, is analytic in $\lambda$, continuous in $x$, and non-vanishing at $x=b$. Because of the homogeneity of (25a) this solution may further be made to satisfy the condition

$$
u(b)=\bar{u}(b) .
$$

We may conclude, then, in a manner entirely analogous to that of $\S 28$, that the function

$$
z(x)=\lambda^{n-2} e^{-\lambda b}\{u(x)-\tilde{u}(x)\}
$$

satisfies an equation of the type (32) and, since it vanishes at $x=b$, is of the form $\epsilon(x, \lambda)$. From this it follows further in the manner of $\S 28$ that there exists a solution of equation (25a) which, for $|\lambda|>N, \sigma \geqq-M$, is analytic in $\lambda$ and continuous in $x$, and which is of the form

$$
u(x)=\bar{u}(x)+\frac{\epsilon(x, \lambda) e^{\lambda b}}{\lambda^{n-2}} .
$$

Inasmuch as zero is a particular function of the type $\epsilon(x, \lambda)$ the results above may be summarized as follows.

Theorem 6. If the complex parameter $\lambda$ is restricted to a region bounded by a line parallel to the axis of imaginaries and exterior to a circle sufficiently large and with center at $\lambda=0$, then the integro-differential equation (25a) admits of a solution of the form

$$
\begin{aligned}
u(x)= & e^{\lambda x}\left\{1+\frac{\gamma_{1}(x)}{\lambda}+\cdots+\frac{\gamma_{n-2}(x)}{\lambda^{n-2}}\right\} \\
& +e^{\lambda b}\left\{\frac{\beta_{1}(x)}{\lambda}+\cdots+\frac{\beta_{n-2}(x)}{\lambda^{n-2}}+\frac{\epsilon(x, \lambda)}{\lambda^{n-2}}\right\} \\
& +e^{\lambda a}\left\{\frac{\alpha_{1}(x)}{\lambda}+\cdots+\frac{\alpha_{n-2}(x)}{\lambda^{n-2}}+\frac{\epsilon(x, \lambda)}{\lambda^{n-2}}\right\} .
\end{aligned}
$$

This solution is analytic in $\lambda$ and continuous in $x$. 
30. The associated equation. Clearly the analysis applied above to the equation (25a) and its formal solution (40), may be applied equally well to the associated equation $(\overline{25 a})$ and its formal solution $(\overline{4} \overline{0})$. In this manner it is found that when $\lambda$ is confined to any right or left-hand half-plane and $|\lambda|>N$, the equation $(\overline{25 a})$ admits of a solution $v(x)$ which is continuous in $x$, analytic in $\lambda$, and of the form

$$
\begin{aligned}
v(x) & =e^{-\lambda x}\left\{1-\frac{\gamma_{1}(x)}{\lambda}+\cdots+(-1)^{n-2} \frac{\bar{\gamma}_{n-2}(x)}{\lambda^{n-2}}\right\} \\
& +e^{-\lambda b}\left\{-\frac{\bar{\beta}_{1}(x)}{\lambda}+\cdots+(-1)^{n-2} \frac{\bar{\beta}_{n-2}(x)}{\lambda^{n-2}}+\frac{\epsilon(x, \lambda)}{\lambda^{n-2}}\right\} \\
& +e^{-\lambda a}\left\{-\frac{\bar{\alpha}_{1}(x)}{\lambda}+\cdots+(-1)^{n-2} \frac{\bar{\alpha}_{n-2}(x)}{\lambda^{n-2}}+\frac{\epsilon(x, \lambda)}{\lambda^{n-2}}\right\} .
\end{aligned}
$$

We remark lastly that since the coefficients and functions involved in equation (25a) and $(\overline{25 a})$ are all real, conjugate imaginary values of $\lambda$ will correspond to the conjugate imaginary solutions $u(x)$ or $v(x)$. Thus if we denote by $\lambda_{c}, u_{c}(x)$, and $v_{c}(x)$ the conjugates of $\lambda, u(x)$, and $v(x)$ respectively,

$$
\begin{gathered}
u\left(x, \lambda_{c}\right)=u_{c}(x, \lambda), \\
v\left(x, \lambda_{c}\right)=v_{c}(x, \lambda) .
\end{gathered}
$$

\section{Chapter 8. The characteristic values}

31. The characteristic equation. The immediately preceding sections have been concerned with the form of a solution of equation (25a). By Chapters 3 and 4 such a solution solves the given normalized integral equation if and only if equation (25b) is also satisfied. This imposes a restriction on the choice of $\lambda$ which we proceed to consider.

Substituting for the factor $\lambda u(t)$ in $(25 \mathrm{~b})$ its value as the second term of (25a) we obtain the equation

$$
L(u)=\int_{a}^{b} V(t)\left\{u^{\prime}(t)-L(u) \varphi(t)-\int_{a}^{b} f(t, \xi) u(\xi) d \xi\right\} d t
$$

which, if we integrate by parts the term involving $u^{\prime}(t)$, takes the form

$$
k_{1} u(a)-k_{2} u(b)+\int_{a}^{b} \Delta \cdot \bar{\varphi}(\xi) u(\xi) d \xi
$$


In this, $\Delta \cdot \bar{\varphi}(\xi)$ is given by formula (26a), and

$$
\begin{aligned}
& k_{1}=V(a)+\mu\left\{1+\int_{a}^{b} V(\xi) \varphi(\xi) d \xi\right\}, \\
& k_{2}=V(b)-\nu\left\{1+\int_{a}^{b} V(\xi) \varphi(\xi) d \xi\right\} .
\end{aligned}
$$

It is convenient to set

$$
\begin{aligned}
& \Omega_{1}(\xi)=\frac{-\Delta \cdot \bar{\varphi}(\xi)}{2 k_{1}}, \\
& \Omega_{2}(\xi)=\frac{\Delta \cdot \bar{\varphi}(\xi)}{2 k_{2}},
\end{aligned}
$$

and to write the equation in the form

$$
k_{1}\left[u(a)-\int_{a}^{b} \Omega_{1}(\xi) u(\xi) d \xi\right]-k_{2}\left[u(b)-\int_{a}^{b} \Omega_{2}(\xi) u(\xi) d \xi\right]=0 .
$$

We shall call this equation the characteristic equation. Its roots, if such exist, are values of $\lambda$ for which the solution of the integro-differential equation satisfies also the given integral equation. We shall call these roots characteristic values and denote them by $\lambda_{i}$.

We observe that since the coefficients of equation (45) are all real, the equation is essentially unchanged if $u(x)$ is replaced by its conjugate imaginary $u_{c}(x)$. From this it follows, by $\S 30$, that the characteristic values occur in conjugate pairs.

Now for $\lambda$ in any right or left hand half-plane we have by Theorem 6 a solution

$$
u(x)=e^{\lambda x}+e^{\lambda b_{\epsilon}}(x, \lambda)+e^{\lambda a_{\epsilon}}(x, \lambda),
$$

which is analytic in $\lambda$ for $|\lambda|>N$. The substitution of this in (45) yields for the characteristic equation the form

$$
\left[k_{1}+\epsilon(\lambda)\right] e^{\lambda a}-\left[k_{2}+\epsilon(\lambda)\right] e^{\lambda b}=0,
$$

in which the functions $\epsilon(\lambda)$ are analytic for $|\lambda|>N$.

32. A further condition on the given equation. It is essential to the procedure which is to be followed in solving equation (46) that neither of the coefficients $k_{1}$ and $k_{2}$ shall vanish. Since $k_{1}$ and $k_{2}$ are seen from the formulas to be rational functions of $\mu$ and $\nu$ we may choose $\mu$ and $\nu$ subject to previous restrictions so that $k_{1} k_{2} \neq 0$, provided only that neither $k_{1}$ nor $k_{2}$ vanishes 
identically in $\mu$ and $\nu$. We shall suppose then that the given integral equation is such that

$$
k_{1}(\mu, \nu) \not \equiv 0, \quad k_{2}(\mu, \nu) \not \equiv 0 .
$$

From the formulas for $k_{1}$ and $k_{2}$ it follows immediately that

$$
\nu k_{1}(\mu, \nu)+\mu k_{2}(\mu, \nu)=\Delta(\mu, \nu) .
$$

It is a clear from this that a sufficient condition for (vi) is that

$$
\Delta(0, v) \neq 0, \quad D(0, \nu) \neq 0,
$$

and

$$
\Delta(\mu, 0) \neq 0, \quad D(\mu, 0) \neq 0 .
$$

For if these conditions are fulfilled $k_{1}$ and $k_{2}$ are defined for the values $(\mu, 0)$ and $(0, \nu)$ and if either one vanished identically relation (47) would entail a contradiction for $\mu=0$ or for $\nu=0$.

The restriction (vi) is an essential one, for we have in the Volterra equations, i.e. those in which $K(x, \xi) \equiv 0$ in one of the regions $R_{1}$ and $R_{2}$, an example of equations which may satisfy all previous conditions, but to which the subsequent results do not apply. To show that these equations are ruled out by condition (vi) we may proceed as follows.

Let us suppose $K(x, \xi) \equiv 0$ for $\xi>x$. Then $W(a)=\nu$ and $\nu \neq 0$ because of (8). We recall that $E(x, \xi)=K_{\xi}(x, \xi)$, and hence that Fredholm's identity (15) yields the formula

$$
K_{\xi}(a, \xi)+F(a, \xi)=\int_{a}^{b} K_{b}(a, t) F(t, \xi) d t .
$$

Upon an integration by parts the right hand member of this assumes the form

$$
K(a, b) F(b, \xi)-K(a, a+) F(a, \xi)-\int_{a}^{b} K(a, t) F_{b}(t, \xi) d t,
$$

of which the first two terms reduce because of $(17 b)$ and (12) to $F(a, \xi)$. Hence we may write

$$
K_{\xi}(a, \xi)=-\int_{a}^{b} K(a, t) F_{t}(t, \xi) d t
$$

or, upon substituting from relation (10),

$$
\int_{0}^{b V(t) F_{b}(t, \xi) d t} \frac{1}{\Delta}=\frac{1}{W(a)}\left[K_{\xi}(a, \xi)+\int_{a}^{b} F_{t}(t, \xi) K(a, t) d t\right]-\frac{V^{\prime}(\xi)}{\Delta} .
$$


Multiplying this by $W(\xi)$ and integrating we obtain the form

$$
\begin{aligned}
\int_{a}^{b} V(t) \int_{a}^{b} F_{t}(t, \xi) \frac{W(\xi)}{\Delta} d \xi d t & =\frac{1}{W(a)} \int_{a}^{b} W(\xi)\left[K_{\xi}(a, \xi)+\int_{a}^{b} F_{t}(t, \xi) K(a, t) d t\right] d \xi \\
& -\frac{1}{\Delta}[W(b) V(b)-W(a) V(a)]+\int_{a}^{b} V(\xi) \frac{W^{\prime}(\xi)}{\Delta} d \xi .
\end{aligned}
$$

With the use of formulas (11) and a rearrangement of terms this leads finally to the relation

$$
\begin{aligned}
\int_{a}^{b} V(t) \varphi(t) d t= & \frac{V(b)-W(a)}{\nu} \\
& -\frac{1}{W(a)}-\int_{a}^{b} W(\xi)\left[K_{\xi}(a, \xi)+\int_{a}^{b} F_{t}(t, \xi) K(a, t) d t\right] d \xi .
\end{aligned}
$$

From this form it is readily found, now, that since $K(x, \xi) \equiv 0$ for $\xi>x$, the second term on the right vanishes, with the result that

$$
k_{1}=\mu+\nu K(b, a), \quad k_{2} \equiv 0 .
$$

33. The solution of the characteristic equation. We consider to begin with the related, simpler equation

$$
k_{1} e^{\rho a}-k_{2} e^{\rho b}=0 .
$$

Because of (vi) we may suppose $\mu$ and $\nu$ so chosen that $k_{1} \neq 0, k_{2} \neq 0$, and the equation is easily solvable. Thus $e^{\rho(b-a)}=k_{1} / k_{2}$, and by taking logarithms the roots are found to be

$$
\rho_{m}=\frac{1}{b-a}\left\{2 m \pi i+\log \frac{k_{1}}{k_{2}}\right\} \quad(m=0, \pm 1, \pm 2, \cdots) .
$$

Solving the equation (46) now formally in the same manner we find

$$
\lambda_{m}=\frac{1}{b-a}\left\{2 m \pi i+\log \frac{k_{1}+\epsilon\left(\lambda_{m}\right)}{k_{2}+\epsilon\left(\lambda_{m}\right)}\right\} .
$$

Then inasmuch as in functional form

we have

$$
\log \frac{k_{1}+\epsilon\left(\lambda_{m}\right)}{k_{2}+\epsilon\left(\lambda_{m}\right)}=\log \frac{k_{1}}{k_{2}}+\epsilon\left(\lambda_{m}\right),
$$

$$
\lambda_{m}=\rho_{m}+\epsilon\left(\lambda_{m}\right) .
$$

Consider now the function

$$
\theta(\lambda)=\lambda-\left[\rho_{m}+\epsilon(\lambda)\right],
$$


the function $\epsilon$ being that of (46a). The function $\theta(\lambda)$ is analytic, and if $\delta>0$ be chosen arbitrarily but sufficiently small it will follow that $|\epsilon(\lambda)|<\delta$ for $|\lambda|>N$. If, therefore, a circle $C_{m}$ with radius $\delta$ be drawn about each of the points $\rho_{m}$, then for $|m|$ sufficiently large the point $\rho_{m}+\epsilon(\lambda)$ will lie within the corresponding circle $C_{m}$ whenever $\lambda$ is a point on the circumference. As $\lambda$ describes this circumference, therefore, the argument of $\theta(\lambda)$ increases by $2 \pi$, from which it follows that $\theta(\lambda)=0$ has precisely one root within $C_{m}$. This means if we denote this root by $\lambda_{m}$ that the points $\lambda_{m}$ are represented asymptotically by the points $\rho_{m}$, namely

$$
\lambda_{m}=\rho_{m}+\epsilon_{m},
$$

where $\epsilon_{1}, \epsilon_{2}, \cdots, \epsilon_{m}, \cdots$ is a sequence of constants such that $\lim _{m \rightarrow \infty} \epsilon_{m}=0$. Moreover since the characteristic values occur in conjugate pairs, while $\rho_{m}$ and $\rho_{-m}$ are conjugate, ${ }^{*}$ we conclude that

$$
\lambda_{m}=\bar{\lambda}_{m}
$$

From the significance of $\rho_{m}$ for the given integral equation, and from the fact that by (49) $\rho_{m}$ involves the ratio $k_{1} / k_{2}$, it follows that this ratio is determined by the integral equation alone and so must be independent $\dagger$ of the parameters $\mu$ and $\nu$.

34. A more precise formula for $\lambda_{n}$. In deducing the formula (50) we assumed only that for the given integral equation $n \geqq 2$. If we have at our disposal the existence of derivatives of higher order a formula more precise than (50) may be deduced. We shall assume, now, for the following deductions that for the given integral equation

$$
n \geqq 4 \text {. }
$$

The assumption (vii) insures the existence of a solution $u(x)$ of equation (25a) which is of the form given by (43) for $n=4$. Substituting this solution in (45) and integrating by parts the integrals involving $e^{\lambda \xi} \Omega(\xi)$ we obtain for the characteristic equation the form

$$
e^{\lambda a}\left\{k_{1}+\frac{k_{12}}{\lambda}+\frac{k_{13}+\epsilon(\lambda)}{\lambda^{2}}\right\}-e^{\lambda b}\left\{k_{2}+\frac{k_{22}}{\lambda}+\frac{k_{23}+\epsilon(\lambda)}{\lambda^{2}}\right\}=0,
$$

* It is assumed at this point that $k_{1} / k_{2}$ is positive. If it is negative we may write $\log \left(k_{1} / k_{2}\right)=$ $\pi i+\log \left|k_{1} / k_{2}\right|$, in which case it is evident that $\rho_{m}$ and $\rho_{-m-1}$ and hence also $\lambda_{m}$ and $\lambda_{-m-1}$ are conjugate. To avoid unnecessary complications we shall continue only with the case $k_{1} / k_{2}>0$, the modifications for $k_{1} / k_{2}<0$ being reasonably obvious.

t The example of the appendix illustrates this. 
where the coefficients $k$ are constants and in particular

$$
\begin{aligned}
& k_{12}=k_{1}\left\{\gamma_{1}(a)+\alpha_{1}(a)+2 \Omega_{1}(a)-2 \int_{a}^{b} \Omega_{1}(\xi) \alpha_{1}(\xi) d \xi\right\}-k_{2} \alpha_{1}(b), \\
& k_{22}=k_{2}\left\{\gamma_{1}(b)+\beta_{1}(b)-2 \Omega_{2}(b)-2 \int_{a}^{b} \Omega_{2}(\xi) \beta_{1}(\xi) d \xi\right\}-k_{1} \beta_{1}(a) .
\end{aligned}
$$

Solving this equation formally in the fashion of $\S 33$ we obtain

$$
\lambda_{m}=\rho_{m}+\frac{1}{b-a} \log \frac{1+\frac{k_{12}}{k_{1} \lambda_{m}}+\frac{k_{13}+\epsilon\left(\lambda_{m}\right)}{k_{1} \lambda_{m}^{2}}}{1+\frac{k_{22}}{k_{2} \lambda_{m}}+\frac{k_{23}+\epsilon\left(\lambda_{m}\right)}{k_{2} \lambda_{m}^{2}}},
$$

or, upon expanding the logarithm as we may, because of (50), for $|m|$ sufficiently large, the form

$$
\lambda_{m}=\rho_{m}+\frac{1}{b-a}\left[\frac{\frac{k_{12}}{k_{1}}-\frac{k_{22}}{k_{2}}}{\lambda_{m}}\right]+\frac{A^{\prime}+\epsilon\left(\lambda_{m}\right)}{\lambda_{m}^{2}},
$$

$A^{\prime}$ representing a constant independent of $m$. From the formulas (46a) and (49) we find readily, however, that

$$
\frac{1}{\lambda_{m}}=\frac{b-a}{2 m \pi i}+\frac{A^{\prime \prime}+\epsilon(m)}{m^{2}},
$$

$A^{\prime \prime}$ being a constant. If this is substituted in the right hand member of the preceding expression we obtain a result which is expressed in the second part of the following theorem.

ThEOREM 7. If the kernel of the normalized integral equation (5) satisfies the conditions (iii), (iv), (v) and (vi) then there exist infinitely many values of the parameter $\lambda$ for which the integral equation admits of a solution. These characteristic values are of the form

where

$$
\lambda_{m}=\frac{1}{b-a}\left\{2 m \pi i+\log \frac{k_{1}}{k_{2}}\right\}+\epsilon_{m} \quad(m=0, \pm 1, \pm 2, \cdots),
$$

$$
\lim _{|m|+\infty} \epsilon_{m}=0 \text {. }
$$

If the kernel of the integral equation satisfies the conditions (iv), (v), (vi) and (vii), these characteristic values may be determined more precisely by the formula

$$
\lambda_{m}=\frac{1}{b-a}\left\{2 m \pi i+\log \frac{k_{1}}{k_{2}}\right\}+\frac{\left(k_{12} / k_{1}\right)-\left(k_{22} / k_{2}\right)}{2 m \pi i}+\frac{A+\epsilon_{m}}{m^{2}} .
$$


The various constants $k$ are given by formulas (44a) and (51), and $A$ also is a constant independent of $m$.

Obviously if a larger number of derivatives of the kernel of the given equation are at hand the method applied above is applicable to the deduction of a formula still more precise than $(50 \mathrm{a})$.

35. The associated equation. It is not necessary in this case to repeat the discussion for the system $(\overline{2} \overline{5})$. By the classical theory of integral equations the associated equations (5) and $(\overline{5})$ have the same characteristic values. Hence formula (50a) serves both.

\section{Chapter 9. The Characteristic FunCtions}

36. The solutions of the integral equation. The characteristic values $\lambda_{m}$ by their very determination are such that the functions $u\left(x, \lambda_{m}\right)$ satisfy not merely equation (25a) but the entire system (25). As such these functions are also solutions of the given normalized integral equation. For brevity we shall denote them by $u_{m}(x)$, and shall call them characteristic functions.

The form of the characteristic function $u_{m}(x)$ is obviously obtained by substituting the value $\lambda=\lambda_{m}$ in the form $u(x)$ given by formula (43). Dividing this formula by $e^{\lambda a}$ and observing that for $|m|$ sufficiently large $e^{\lambda_{m}(x-a)}$ is uniformly bounded, we obtain because of (vii) the formula

$$
u_{m}(x)=e^{\lambda_{m}(x-a)}\left\{1+\frac{\gamma_{1}(x)}{\lambda_{m}}\right\}+e^{\lambda_{m}(b-a)} \frac{\beta_{1}(x)}{\lambda_{m}}+\frac{\alpha_{1}(x)}{\lambda_{m}}+\frac{H\left(x, \lambda_{m}\right)}{\lambda_{m}{ }^{2}} .
$$

Since by $(50 \mathrm{a})$

$$
\begin{aligned}
e^{\lambda_{m}(x-a)} & =e^{\rho_{m}(x-a)+\left[\left(1 / 2 m x_{i}\right)\left(\left(k_{12} / k_{1}\right)-\left(k_{22} / k_{2}\right)\right)+H(m) / m_{2}\right](x-a)} \\
& =e^{\rho_{m}(x-a)}\left[1+\frac{x-a}{2 m \pi i}\left(\frac{k_{12}}{k_{1}}-\frac{k_{22}}{k_{2}}\right)+\frac{H(x, m)}{m^{2}}\right],
\end{aligned}
$$

we find in terms of $\rho_{m}$ and $m$

$$
u_{m}(x)=e^{\rho_{m}(x-a)}\left\{1+\frac{Q_{1}(x)}{m}\right\}+\frac{Q_{2}(x)}{m}+\frac{H(x, m)}{m^{2}},
$$

where

$$
Q_{1}(x)=\frac{\gamma_{1}(x)(b-a)+\left(\left(k_{12} / k_{1}\right)-\left(k_{22} / k_{2}\right)\right)(x-a)}{2 \pi i}
$$

and

$$
Q_{2}(x)=\frac{b-a}{2 \pi i}\left\{e^{\rho_{m}(b-a)} \beta_{1}(x)+\alpha_{1}(x)\right\}
$$


By (48), however.

$$
e^{\rho_{m}(b-a)}=\frac{k_{1}}{k_{2}}
$$

Hence the function $Q_{2}(x)$ is independent of $m$ and substituting for $\beta_{2}(x)$ and $\alpha_{1}(x)$ their values from formulas (39) we find that

$$
\varrho_{2}(x)=\frac{-(b-a)}{2 \pi i}\left[\mu+\frac{k_{1}}{k_{2}} \nu\right] \varphi(x) .
$$

Deductions precisely similar to those above based on the function $(\overline{4} \overline{3})$ yicld the characteristic functions of equation $(\overline{5})$. We have first

$$
\because n(x)=\cdots \lambda_{m}\left(s \cdots\left\{1-\frac{\gamma_{1}(x)}{\lambda_{m}}\right\}-\lambda_{m} \cdots, \frac{\bar{\beta}_{1}(x)}{\lambda_{m}}-\frac{\overline{\alpha_{1}}(x)}{\lambda_{m}}+\frac{H\left(x, \lambda_{m}\right)}{\lambda_{m}{ }^{2}}\right.
$$

Iroin which in the manner above we obtain

$$
i_{m}(x)=\theta p_{n i}=\left\{1-\frac{\rho_{1}(x)}{m}\right\}+\frac{Q_{3}(x)}{m}+\frac{H(x, m)}{m^{2}} .
$$

In this $Q_{1}(x)$ is the function occurring also in (52), and

$$
\varphi_{3}(x)=\frac{b-a}{2 \pi i}\left[\nu+\mu \frac{k_{2}}{k_{1}}\right] \bar{\varphi}(x) \text {. }
$$

37. The normalized set. By the classical theory of integral equations the set of functions $u_{m}(x), i_{m}(x)$ is biorthogonal, that is,

$$
\int_{a}^{b} u_{m}(x) r_{p}(x) d x=0, \quad m \neq p .
$$

The set of functions is said to be normalized if further

$$
\int_{a}^{h} u_{m}(x) v_{m}(x) d x=1
$$

Forming the product $u_{m}(x) v_{m}(x)$ from formulas (52) and $(\overline{5} \overline{2})$ we obtain

$$
u_{m}(x) i_{m}(x)=1+\frac{Q_{2}(x) e^{-i_{m}(x \cdots \cdots)}+Q_{3}(x) e^{\rho_{m}(x-a)}}{m}+\frac{I I(x, m)}{m^{2}} .
$$

Then since $\left|\rho_{m}\right|$ is of the order $m$, while $Q_{2}(x)$ and $Q_{3}(x)$ are possessed of a derivative, we obtain as the result of an integration by parts

$$
\int_{a}^{b} \frac{Q_{i}(x) e^{ \pm \rho_{m}(x-a)} d x}{m}=\frac{H(m)}{m^{2}} \quad(i=2,3) .
$$


Accordingly

$$
\int_{a}^{b} u_{m}(x) v_{m}(x) d x=(b-a)+\frac{H(m)}{m^{2}} .
$$

We may therefore normalize the solutions given by $(52)$ and $(\overline{5} \overline{2})$ by multiplying them by a suitable factor of the form

$$
\frac{1}{\sqrt{b-a}}+\frac{H(m)}{m^{2}} \text {. }
$$

Thus we obtain the following theorem.

THEOREM 8. If the kernel of the normalized integral equation (5) satisfies the conditions (iv), (v), (vi), and (vii), and the kernel of the associated integral equation satisfies condition $(\overline{\mathrm{v}})$, then the normalized characteristic solutions of these equations are given respectively, for $|m|$ sufficiently large, by the formulas

$$
\begin{aligned}
& u_{m}(x)=\frac{1}{\sqrt{b-a}}\left[e^{\rho_{m}(x-a)}\left\{1+\frac{Q_{1}(x)}{m}\right\}+\frac{Q_{2}(x)}{m}+\frac{H(x, m)}{m^{2}}\right], \\
& v_{m}(x)=\frac{1}{\sqrt{b-a}}\left[e^{-\rho_{m}(x-a)}\left\{1-\frac{Q_{1}(x)}{m}\right\}+\frac{Q_{3}(x)}{m}+\frac{H(x, m)}{m^{2}}\right] .
\end{aligned}
$$

Finally we remark that by $\S 30$ and $\S 33$ the functions $u_{m}(x)$ and $u_{-m}(x)$, as well as the functions $v_{m}(x)$ and $v_{-m}(x)$, are conjugate imaginary.

Chapter 10. The closure of the set of characteristic functions

38. Birkhoff's theorem. It has been shown in the preceding chapters that for $|m|$ sufficiently large the characteristic values of the given integral equation are simple, and that the corresponding biorthogonal set of characteristic functions $u_{m}(x), v_{m}(x)$ may be normalized. We shall now make the further assumption:

(viii) All the characteristic values of the given integral equation are simple.

Then the entire set of solutions $u_{m}(x), v_{m}(x)$ may be normalized. We wish to show that the set is also closed. To this end we shall employ the following theorem which is a generalization of a theorem given by Birkhoff* for orthogonal sets.

* Birkhoff, Proceedings of the National Academy of Sciences, vol. 3 (1917), p. 656. 
THEOREM. If $w_{m}(x), z_{m}(x), m=0, \pm 1, \pm 2, \cdots$, is a closed normalized biorthogonal set of functions, and if $\bar{w}_{m}(x), \bar{z}_{m}(x)$ is a second normalized biorthogonal set, then this second set is also closed provided (a) the series

$$
\begin{aligned}
{\left[w_{0}(x)-\bar{w}_{0}(x)\right] z_{0}(y)+} & \sum_{m=1}^{\infty}\left\{\left[w_{m}(x)-\bar{w}_{m}(x)\right] z_{m}(y)\right. \\
& \left.+\left[w_{-m}(x)-\bar{w}_{-m}(x)\right] z_{-m}(y)\right\}
\end{aligned}
$$

converges to a function $H(x, y)$ less than $1 / 2(b-a)$ in numerical magnitude, and (b) the convergence is such that the series multiplied through by an arbitrary continuous function $f(x)$ can be integrated term by term with respect to $x$, and yields a series which converges uniformly on any closed sub-interval of $a<y<b$, and of which the sum of any number of terms remains uniformly bounded for $a \leqq y \leqq b$.

The proof, except for minor modifications, is that given by Birkhoff. Thus if the set $\bar{w}_{m}(x), \bar{z}_{m}(x)$ is not closed, there exists a function $f(x) \neq 0$ which is continuous on $(a, b)$ and such that

$$
\int_{a}^{b} f(x) \bar{w}_{m}(x) d x=0 \text { for all } m .
$$

In this event if we multiply through the equation of definition for $H(x, y)$ by this $f(x)$ and integrate term by term as we may by hypothesis, we find

$$
\begin{aligned}
\int_{a}^{b} H(x, y) f(x) d x= & \int_{a}^{b} f(x) w_{0}(x) z_{0}(y) d x \\
& +\sum_{m=1}^{\infty} \int_{a}^{b} f(x)\left[w_{m}(x) z_{m}(y)+w_{-m}(x) z_{-m}(y)\right] d x,
\end{aligned}
$$

where the series on the right hand side converges under the hypothesis to a bounded function which is continuous for $a<y<b$. This function on the open interval is precisely $f(y)$. In fact the difference

$$
f(y)-\left\{\int_{a}^{b} f(x) w_{0}(x) z_{0}(y) d x+\sum_{m=1}^{\infty} \int_{a}^{b} f(x)\left[w_{m}(x) z_{m}(y)+w_{-m}(x) z_{-m}(y)\right] d x\right\}
$$

is a function $\varphi(y)$ bounded on $(a, b)$, continuous for $a<y<b$, and such that

$$
\int_{a}^{b} \varphi(y) w_{l}(y) d y=0 \text { for all } l .
$$

This latter property is readily drawn from the fact that the series involved may be integrated term by term, while the set $w_{m}(y), z_{m}(y)$ is normalized 
biorthogonal. But the set $w_{m}(y), z_{m}(y)$ is closed by hypothesis. Hence we infer that $\varphi(y) \equiv 0, a<y<b$, and that the right hand member of the preceding equation has the value $f(y)$. That equation may now be written

$$
\int_{a}^{b} H(x, y) f(x) d x=f(y), \quad a<y<b .
$$

If the upper bound of $|f(x)|$ on $(a, b)$ is $F \neq 0$ we obtain from this since $|H(x, y)|<1 / 2(b-a)$ the relation

$$
|f(y)|<\frac{1}{2} F .
$$

But this is impossible since the left hand member either takes on the value $F$ or comes arbitrarily near to it. The set $\bar{w}_{m}(x), \bar{z}_{m}(x)$ is therefore closed also.

39. The integro-differential system with an additional parameter. We consider now the system

$$
\begin{aligned}
& \text { (a) } \quad u^{\prime}(x)-\lambda u(x)=\eta\left[L(u) \varphi(x)+\int_{a}^{b} f(x, \xi) u(\xi) d \xi\right] \\
& \text { (b) } \quad L(u)=\eta \lambda \int_{a}^{b} V(\xi) u(\xi) d \xi .
\end{aligned}
$$

For $\eta=1$ this is simply the system (25) and defines the set of functions $u_{m}(x, 1) \equiv u_{m}(x)$. For $\eta=0$ it is an ordinary differential system and defines a set $u_{m}(x, 0)$. For both these values of $\eta$ there exists an associated or adjoint system with its corresponding set of solutions $v_{m}(x, 1)$ or $v_{m}(x, 0)$ which is biorthogonal to the respective set above. It is our purpose to show that for other suitable values of $\eta$ in the circle $C:|\eta| \leqq 1$ of the complex $\eta$ plane the system (55) also defines a set of functions $u_{m}(x, \eta)$ and that there exists a biorthogonal normal set $v_{m}(x, \eta)$. Subsequently it will be shown that the repeated application of Birkhoff's theorem makes it possible to conclude the closure of the set $u_{m}(x, 1), v_{m}(x, 1)$ from the known closure of the set $u_{m}(x, 0)$, $v_{m}(x, 0)$.

The system (55) will be equivalent to an integral equation of the form (5) provided the hypotheses of Chapter 4 as applied to system (55) are met. The extension of the deductions of Chapter 4 to system (55) is made formally by replacing respectively $\varphi(x), f(x, \xi)$, and $V(\xi)$, by $\eta \varphi(x), \eta f(x, \xi)$ and $\eta V(\xi)$. This is readily found to result in replacing $F(x, \xi)$ by $\eta F(x, \xi)$, and $\Phi(x)$ by $\Phi(x, \eta)=\frac{1}{2}(1-\eta)+\eta \Phi(x)$. The essential hypothesis to be met is that the function $\eta F(x, \xi)$ be possessed of a reciprocal function $E(x, \xi, \eta)$. By the classical theory of integral equations this reciprocal is a meromorphic function 
of $\eta$. Hence its poles within the circle $C$ are finite in number and may be denoted by $\eta_{i}$. Then for $\eta$ in any closed sub-region of $C$, call it $C^{\prime}$, which excludes these points $\eta_{i}$, the function $E(x, \xi, \eta)$ is analytic, and by Theorem 4 the system (55) is equivalent to an integral equation

$$
u(x, \eta)=\lambda \int_{a}^{b} K(x, \xi, \eta) u(\xi, \eta) d \xi .
$$

The kernel $K(x, \xi, \eta)$, since it satisfies the relation (31) with the formal modifications noted above, is readily found to be continuous in $x$ and $\eta$, and in fact analytic in $\eta$ in the region $C^{\prime}$.

Consider now the associated equation

$$
v(x, \eta)=\lambda \int_{a}^{b} K(\xi, x, \eta) v(\xi, \eta) d \xi,
$$

and the deductions of Chapter 3 as applied to it. From the analytic character of $K(\xi, x, \eta)$ and its relation to the kernel of $(56)$, the function $\bar{E}(x, \xi, \eta)$ of $\S 11$ is readily found to be analytic in $\eta$. From the classical theory of integral equations we draw again the fact that $\bar{E}(x, \xi, \eta)$ possesses a reciprocal $\bar{F}(x, \xi, \eta)$ which is meromorphic in $\eta$. If we add to the set of points $\eta_{i}$ those poles of $\bar{F}(x, \xi, \eta)$ which lie within $C^{\prime}$ and denote by $C^{\prime \prime}$ any closed sub-region of $C^{\prime}$ which excludes also these values $\eta_{i}$, it follows that $\bar{F}(x, \xi, \eta)$ exists and is analytic in $C^{\prime \prime}$. By Chapter 3 it follows then that the equation $(\overline{56})$ is also equivalent to an integro-differential system which is the associated system of (55).

It is essential to observe that in obtaining this result we have not excluded from $C^{\prime \prime}$ either $\eta=1$ or $\eta=0$. The case $\eta=1$ is covered by the hypotheses of the preceding chapters. For $\eta=0$ the system (55) is equivalent to the integral equation

$$
u(x, 0)=\lambda \int_{a}^{b} G(x, \xi) u(\xi, 0) d \xi,
$$

and the associated equation

is equivalent to the system

$$
v(x, 0)=\lambda \int_{a}^{b} G(\xi, x) v(\xi, 0) d \xi
$$

$$
\begin{gathered}
v^{\prime}(x, 0)+\lambda v(x, 0)=0, \\
\bar{L}(v)=0 .
\end{gathered}
$$

The extension of the deductions of Chapters 5, 6, and 7 to the system (55) and its associated system may be made now with the formal modifica- 
tions noted. For the deductions of Chapters 8 and 9 the condition (vi), which in the present case takes the form

$$
\begin{aligned}
& k_{1}(\eta)=\eta V(a)+\mu\left[1+\eta^{2} \int_{a}^{b} V(\xi) \varphi(\xi) d \xi\right] \neq 0, \\
& k_{2}(\eta)=\eta V(b)-\nu\left[1+\eta^{2} \int_{a}^{b} V(\xi) \varphi(\xi) d \xi\right] \neq 0,
\end{aligned}
$$

must be met. For $\eta=1$ this is assured by previous hypotheses. Hence $k_{1}$ and $k_{2}$ as functions of $\eta$ are not identically zero, and there are at most four values of $\eta$ for which either $k_{1}$ or $k_{2}$ will vanish. Let such of these values as lie in $C^{\prime \prime}$ be added to the set of points $\eta_{i}$ and denote by $C^{\prime \prime \prime}$ any closed subregion of $C^{\prime \prime}$ which excludes also these points. This does not exclude $\eta=1$, and if we suppose, as we may and shall, that $\mu \neq 0, \nu \neq 0$, it does not exclude $\eta=0$. For $\eta$ in the region $C^{\prime \prime \prime}$, then, the biorthogonal set of functions $u_{m}(x, \eta), v_{m}(x, \eta)$ are for $|m|$ sufficiently large, normalized in the form

$$
\begin{array}{r}
u_{m}(x, \eta)=\frac{1}{\sqrt{b-a}}\left[e^{2 m \pi i \frac{x-a}{b-a}+(x-a) \theta(\eta)}\left\{1+\frac{Q_{1}(x, \eta)}{m}\right\}\right. \\
\left.+\frac{Q_{2}(x, \eta)}{m}+\frac{H(x, \eta, m)}{m^{2}}\right], \\
v_{m}(x, \eta)=\frac{1}{\sqrt{b-a}}\left[e^{-2 m \pi i \frac{x-a}{b-a}-(x-a) \theta(\eta)}\left\{1-\frac{Q_{1}(x, \eta)}{m}\right\}\right. \\
\left.+\frac{Q_{3}(x, \eta)}{m}+\frac{H(x, \eta, m)}{m^{2}}\right],
\end{array}
$$

where

$$
\theta(\eta)=\frac{1}{b-a} \log \frac{k_{1}(\eta)}{k_{2}(\eta)},
$$

and the functions $Q_{1}(x, \eta), Q_{2}(x, \eta), Q_{3}(x, \eta), H(x, \eta, m)$ are found from formulas (53), (39), and an analysis of the deductions of Chapters 7, 8 and 9 to be continuous in $x$ and $\eta$ for $\eta$ in the region $C^{\prime \prime \prime}$.

It remains to consider the functions of the set $u_{m}(x, \eta), v_{m}(x, \eta)$ for small index $m$. Let us denote by $D(\eta, \lambda)$ the Fredholm determinant for the kernel of equation (56). By the classical theory of integral equations this determinant is analytic in $\lambda$ and $\eta$ for $\eta$ in the region $C^{\prime \prime \prime}$, and the characteristic values of equation (56) are the roots $\lambda=\lambda(\eta)$ of the equation

$$
D(\eta, \lambda)=0 \text {. }
$$

For $\eta=1$ the roots of this equation are all simple by the hypothesis (viii), 
and for $\eta=0$ they may be explicitly determined from system (55) and are also found to be simple. It follows* that the equations

$$
\begin{aligned}
D(\eta, \lambda) & =0, \\
\frac{\partial D(\eta, \lambda)}{\partial \lambda} & =0
\end{aligned}
$$

admit of common roots, which will of course be multiple roots of (58), only for isolated values of $\eta$. If we add such of these values as lie in $C^{\prime \prime \prime}$ to the set $\eta_{i}$, then for $\eta$ in any closed sub-region of $C^{\prime \prime \prime}$, call it $C^{\mathrm{IV}}$, which excludes also these values, the roots of (58) are all simple and are analytic in $\boldsymbol{\eta}$. We observe that by the remarks above $\eta=0$ and $\eta=1$ are not excluded from $C^{\text {Iv }}$. We shall designate by $\lambda_{m}(\eta)$ that root of (58) which joins analytically with $\lambda_{m}(0)$. It is easily seen that for large values of $|m|$ this agrees with the notation previously adopted in Chapter 8. It follows from the simplicity of the values $\lambda_{m}(\eta)$ that the entire biorthogonal set $u_{m}(x, \eta), v_{m}(x, \eta)$ may be considered normalized. Likewise it is readily found that the functions $u_{m}(x, \eta), v_{m}(x, \eta)$ are continuous in $x$ and $\eta$.

40. A change of variable. The set $u_{m}(x, \eta), v_{m}(x, \eta)$ is not directly adaptable to the application of Birkhoff's theorem. For this reason we introduce the set $w_{m}(x, \eta), z_{m}(x, \eta)$ defined by the relations

$$
\begin{gathered}
w_{m}(x, \eta)=u_{m}(x, \eta) e^{-(x-a) \theta(\eta)}, \\
z_{m}(x, \eta)=v_{m}(x, \eta) e^{(x-a) \theta(\eta)} .
\end{gathered}
$$

The points $\eta_{i}$ in the circle $C$ form a finite set which includes neither $\eta=0$ nor $\eta=1$. The constant $r>0$ may be so chosen, therefore, that of the circles drawn with radii $r$ and centers $\eta_{i}$ no two have a point in common and none includes the point $\eta=0$ or $\eta=1$. We shall suppose such circles drawn and their interiors removed from the circle $C$. The remaining region is of the type denoted by $C^{\mathrm{IV}}$ and in this region we may draw a curve $\Gamma$ of finite length connecting $\eta=0$ with $\eta=1$. For $\eta$ on the curve $\Gamma$, then, $\theta(\eta)$ is analytic and therefore bounded. Inasmuch as $w_{m}(x, \eta) z_{p}(x, \eta)=u_{m}(x, \eta) v_{p}(x, \eta)$, it follows that the set $w_{m}(x, \eta), z_{m}(x, \eta)$ is also biorthogonal and normal. From formulas (57) we obtain readily

$$
\begin{aligned}
& w_{m}(x, \eta)=\frac{1}{\sqrt{b-a}}\left[e^{2 m \pi i \frac{x-a}{b-a}}\left\{1+\frac{Q_{1}(x, \eta)}{m}\right\}+\frac{Q_{2}{ }^{\prime}(x, \eta)}{m}+\frac{B(x, \eta, m)}{m^{2}}\right] \\
& z_{m}(x, \eta)=\frac{1}{\sqrt{b-a}}\left[e^{-2 m \times i_{i}^{x-a}}\left\{1-\frac{Q_{1}(x, \eta)}{m}\right\}+\frac{Q_{3}^{\prime}(x, \eta)}{m}+\frac{B(x, \eta, m)}{m^{2}}\right],
\end{aligned}
$$

* Forsyth, A. R., Theory of Functions of two Complex Variables, Cambridge University Press, 1914, pp. 206-209. 
in which the functions $Q_{1}, Q_{2}{ }^{\prime}, Q_{3}{ }^{\prime}$ and $H$ are continuous in $x$ and $\eta$, and therefore uniformly continuous for $\eta$ on $\Gamma$ and $x$ on the interval $(a, b)$.

41. The application of Birkhoff's theorem. From formulas (57a) we obtain now

where

$$
w_{m}(x, \eta)-w_{m}(x, \bar{\eta})=\frac{1}{b-a}\left[e^{2 m \pi i \frac{x-a}{b-a}} \frac{\delta Q_{1}(x)}{m}+\frac{\delta Q_{2}^{\prime}(x)}{m}+\frac{\delta H(x, m)}{m^{2}}\right]
$$

It follows then that

$$
\delta Q(x)=Q(x, \eta)-Q(x, \bar{\eta}), \quad \text { etc. }
$$

$$
\begin{aligned}
& {\left[w_{0}(x, \eta)-w_{0}(x, \bar{\eta})\right] z_{0}(y, \eta)+\sum_{m=1}^{\infty}\left[w_{m}(x, \eta)-w_{m}(x, \bar{\eta})\right] z_{m}(y, \eta)} \\
& +\left[w_{-m}(x, \eta)-w_{-m}(x, \bar{\eta})\right] z_{-m}(y, \eta) \\
& =\sum_{m=-M+1}^{M-1}\left[w_{m}(x, \eta)-w_{m}(x, \bar{\eta})\right] z_{m}(y, \eta) \\
& +\frac{\delta Q_{1}(x)}{b-a} \sum_{m=M}^{\infty} \frac{e^{2 m \pi i \frac{x-y}{b-a}}-e^{-2 m \pi i \frac{x-y}{b-u}}}{m} \\
& +\frac{\delta Q_{2}^{\prime}(x)}{b-a} \sum_{m=M}^{\infty} \frac{e^{-2 m \pi i \frac{y-a}{b-a}}-e^{2 m \pi i} \frac{y=a}{b-a}}{m} \\
& +\frac{1}{b-a} \sum_{m=M}^{\infty} \frac{\delta H(x, m) \cdot H(y, \eta, m)}{m^{2}} .
\end{aligned}
$$

Let us consider separately each of the series occurring on the right of this expression. The first series is a finite sum which is small in numerical value for $\eta$ and $\bar{\eta}$ on $\Gamma$ and $|\eta-\bar{\eta}|$ small. Also since $H(x, \eta, m)$ is uniformly bounded in $m$, and is uniformly continuous in $x$ and $\eta$ for $\eta$ on $\Gamma$ and $x$ on $(a, b)$, it follows that the last series converges uniformly in $x$ and $y$ to a value which is numerically small for $|\eta-\bar{\eta}|$ small.

The second and third series may be written respectively in the forms

$$
\begin{array}{r}
2 i \frac{\delta Q_{1}(x)}{b-a} \sum_{m=M}^{\infty} \frac{\sin 2 m \pi\left(\frac{x-y}{b-a}\right)}{m}, \\
-2 i \frac{\delta Q_{2}{ }^{\prime}(x)}{b-a} \sum_{m=M}^{\infty} \frac{\sin 2 m \pi\left(\frac{y-a}{b-a}\right)}{m} .
\end{array}
$$


Now series of the type $\Sigma[(\sin m z) / m]$ are known to converge uniformly save in the immediate neighborhood of $z=0, \pm 2 \pi, \pm 4 \pi, \cdots$, where, however, the sum of any number of terms remains uniformly bounded.* It follows that each of the series in question here converges to a value numerically small because of the uniform smallness of the coefficients $\delta Q_{1}(x), \delta Q_{2}^{\prime}(x)$ for $|\eta-\bar{\eta}|$ small. Moreover when multiplied through by a continuous function of $x$ the series may be integrated term by term with respect to $x$, and will yield in the case of the former a series which converges uniformly for $a \leqq y \leqq b$, and in the case of the latter a series which converges uniformly on any closed sub-interval of $a<y<b$, and of which the sum of any number of terms remains bounded for $a \leqq y \leqq b$. It is evident, therefore, that for a properly chosen constant $\delta>0$ and $|\eta-\bar{\eta}| \leqq \delta, \eta, \bar{\eta}$ on $\Gamma$, the convergence of the series (59) fulfills the hypotheses of Birkhoff's theorem. We may conclude, therefore, that the set of functions $w_{m}(x, \bar{\eta}), z_{m}(x, \bar{\eta})$ is closed if the set $w_{m}(x, \eta), z_{m}(x, \eta)$ is closed.

Let the curve $\Gamma$ be divided now by points $\eta^{(j)}$ such that

$$
\eta^{(0)}=0,\left|\eta^{(j)}-\eta^{(j-1)}\right| \leqq \delta
$$

and let the number of divisions be $s$, so that $\eta^{(\theta)}=1$. For $\eta^{(0)}$ we have the set of functions

$$
w_{m}(x, 0)=\frac{1}{\sqrt{b-a}} e^{2 m \pi i \frac{x-a}{b-a}}, \quad z_{m}(x, 0)=\frac{1}{\sqrt{b-a}} e^{-2 m \pi i \frac{x-a}{b-a}},
$$

which is known to be closed. By an application of Birkhoff's theorem we may conclude then that the set $w_{m}\left(x, \eta^{(j)}\right), z_{m}\left(x, \eta^{(j)}\right)$ is closed for $j=1$, and by successively repeated applications for $j=2,3, \cdots, s$. This last application proves the closure of the set $w_{m}(x, 1), z_{m}(x, 1)$. This, however, implies that the relation

$$
\int_{a}^{b} u_{m}(x) f(x) d x=\int_{a}^{b} w_{m}(x, 1) e^{\frac{x-a}{b-a} \log \frac{k_{1}}{k_{2}}} f(x) d x=0
$$

can be true for all $m$ only if

$$
e^{\frac{x-a}{b-a} \log \frac{k_{1}}{k_{2}}} f(x) \equiv 0
$$

i.e. if $f(x) \equiv 0$. It follows from this, however, that the set $u_{m}(x), v_{m}(x)$ is closed. This is the closure we wished to establish and hence we have the following theorem:

- Cf., e. g., Jackson, D., Rendiconti del Circolo Matematico di Palermo, vol. 32 (1911), pp. 257-262, and Bocher, M., Annals of Mathematics, ser. 2, vol. 7 (1906), pp. 110, 111. 
THEOREM 9. If the kernel of the given integral equation satisfies the conditions (iv)-(viii), and that of the associated integral equation satisfies condition $(\bar{v})$, then the set of characteristic solutions $u_{m}(x), v_{m}(x)$ is closed.

Chapter 11. The nON-existence of other characteristic VALUES OR FUNCTIONS

42. The possibilities of omission. The deduction of a solution of equation (25a), and hence also the deduction of the characteristic values, was based on the methods of Chapter 6. It is conceivable that other methods might yield a different form of solution, from which either different characteristic values or different characteristic functions or both might result. We shall consider in turn the following questions:

(a) May there exist a characteristic value $\bar{\lambda}$ and a corresponding solution $\bar{u}(x) \neq 0$ which are not included in the sets $\lambda_{m}$ and $u_{m}(x)$ ?

(b) May there exist a characteristic function $\bar{u}(x) \neq 0$ which corresponds, say, to $\lambda_{l}$, but is not identical with the $u_{l}(x)$ found?

(c) May the characteristic function $u_{l}(x)$ found correspond also to a characteristic value $\bar{\lambda}$ not identical with $\lambda_{l}$ ?

Case (a). Suppose the value $\bar{\lambda}$ and the function $\bar{u}(x)$ mentioned under (a) above to exist. From the biorthogonality of the solutions of associated integral equations it follows that

$$
\int_{a}^{b} \bar{u}(x) v_{m}(x) d x=0 \quad(m=0, \pm 1, \pm 2, \cdots) .
$$

Since the set $u_{m}(x), v_{m}(x)$ is closed, however, this demands $\bar{u}(x) \equiv 0$.

Case (b). Suppose $\bar{u}(x)$ is a solution which corresponds to the value $\lambda_{l}$. Then from the biorthogonality we conclude that

$$
\int_{a}^{b} \bar{u}(x) v_{m}(x) d x=0, \quad m \neq l .
$$

If for $m=l$ the integral also vanishes we may conclude as above from the closure of the set $u_{m}(x), v_{m}(x)$, that $\bar{u}(x) \equiv 0$. If this is not the case we may suppose the solution $\bar{u}(x)$ normalized by multiplication with a suitable constant so that

Then, however,

$$
\int_{a}^{b} \bar{u}(x) v_{l}(x) d x=1
$$

$$
\int_{a}^{b}\left\{\bar{u}(x)-u_{l}(x)\right\} v_{m}(x) d x=0 \quad(m=0, \pm 1, \cdots),
$$


and from the closure of the set $u_{m}(x), v_{m}(x)$ it follows that

$$
\bar{u}(x) \equiv u_{l}(x) .
$$

Case (c). Suppose that the function $u_{l}(x)$ corresponds to a characteristic value $\lambda \neq \lambda_{l}$. We have in this case

$$
\begin{gathered}
u_{l}(x)=\lambda_{l} \int_{a}^{b} K(x, \xi) u_{l}(x) d x, \\
u_{l}(x)=\bar{\lambda} \int_{a}^{b} K(x, \xi) u_{l}(x) d x,
\end{gathered}
$$

from which, since $\bar{\lambda}-\lambda_{l} \neq 0$,

$$
\int_{a}^{b} K(x, \xi) u_{l}(\xi) d \xi=0
$$

This, however, is impossible since $u_{l}(x)$ is a solution of the given integral equation and $u_{l}(x) \neq 0$.

It is seen, therefore, that there exist no characteristic values or functions other than those determined in Chapters 8 and 9.

Chapter 12. The EXPansion of an ARbitrary function

43. The related differential system. The method by which the properties of the solutions $u_{m}(x)$ for the expansion of an arbitrary function are to be deduced is based on a suitable comparison of the set $u_{m}(x)$ with the set of solutions $y_{m}(x)$ of the differential system

$$
\begin{aligned}
y^{\prime}(x)-\rho y(x) & =0, \\
k_{1} y(a)-k_{2} y(b) & =0 .
\end{aligned}
$$

This system is related to the given integral equation in that the constants $k_{1}$ and $k_{2}$ are those which occur also in the characteristic equation (45).

It is essential, therefore, to have at hand the expansion theorem for a system of type (60). In a form which may be found by specialization of results obtained by the author* for more general differential systems this theorem may be stated as follows:

- Developments associated with a boundary problem not linear in the parameter, these Transactions, vol. 25 (1923), pp. 155-172. If in the theorem there stated (p. 171) we set $n=1, a_{1}(x)=1$ and change the dependent variable and the parameter respectively by the substutitions

the system considered takes the form (60) above.

$$
u(x)=\sqrt{b-a} k_{1} y(x) e^{\frac{x-a}{b-a} \log \frac{k_{2}}{k_{1}}}, \quad \rho=\frac{1}{\lambda-\alpha_{1}}-\frac{1}{b-a} \log \frac{k_{2}}{k_{1}},
$$


If $f(x)$ is any function which on the interval $(a, b)$ consists of at most a finite number of pieces, each real and continuous and having a continuous derivative, then $f(x)$ may be expanded in a series of the form

$$
f(x) \sim \sum_{m=-\infty}^{+\infty} \int_{a}^{b} f(t) z_{m}(t) d t \cdot y_{m}(x),
$$

where the functions $y_{m}(x)$ and $z_{m}(x)$ are respectively the normalized solutions of system (60) above and its adjoint system. This series will converge to $\frac{1}{2}\{f(x+)+f(x-)\}$ for $a<x<b$, to $\left(1 / 2 k_{1}\right)\left\{k_{1} f(a+)+k_{2} f(b-)\right\}$ for $x=a$, and to $\left(1 / 2 k_{2}\right)\left\{k_{1} f(a+)+k_{2} f(b-)\right\}$ for $x=b$.

44. The function $\varphi(x, \xi, p)$. We consider now the function $\varphi(x, \xi, p)$ defined by the relation

$$
\varphi(x, \xi, p)=\sum_{m=-p}^{p}\left[u_{m}(x) v_{m}(\xi)-y_{m}(x) z_{m}(\xi)\right] .
$$

Its explicit form may be obtained from the solution of system (60), which yields

$$
y_{m}(x) z_{m}(\xi)=\frac{1}{b-a} e^{\rho_{m}(x-\xi)},
$$

and the formulas (54). It is found in this way that for $|m|$ sufficiently large

$$
\begin{gathered}
u_{m}(x) v_{m}(\xi)-y_{m}(x) z_{m}(\xi)=\frac{1}{b-a}\left\{\frac{Q_{1}(x)-Q_{1}(\xi)}{m} e^{\rho_{m}(x-\xi)}+\frac{Q_{2}(x)}{m} e^{-\rho_{m}(\xi-a)}\right. \\
\left.+\frac{H(x, m) H(\xi, m)}{m^{2}}+\frac{Q_{3}(x) e^{\rho_{m}(x-a)}}{m}\right\} .
\end{gathered}
$$

With the use, now, of the notation of Chapter 10, i.e.

$$
\rho_{m}=\frac{2 m \pi i}{b-a}+\theta, \quad \theta=\frac{1}{b-a} \log \frac{k_{2}}{k_{2}},
$$

and with the choice of a number $M$ fixed and sufficiently large, we obtain the formula

$$
\varphi(x, \xi, p)=\varphi(x, \xi, M)+\sum_{i=1}^{4} \psi_{i}(x, \xi, p)
$$


where

$$
\begin{aligned}
& \psi_{1}(x, \xi, p)=\frac{2 i}{b-a}\left\{Q_{1}(x)-Q_{1}(\xi)\right\} e^{\theta(x-\xi)} \sum_{m=M+1}^{p} \frac{\sin 2 m \pi\left(\frac{x-\xi}{b-a}\right)}{m}, \\
& \psi_{2}(x, \xi, p)=\frac{-2 i}{b-a} Q_{2}(x) e^{-\theta(\xi-a)} \sum_{m=M+1}^{p} \frac{\sin 2 m \pi\left(\frac{\xi-a}{b-a}\right)}{m} \\
& \psi_{2}(x, \xi, p)=\sum_{m=M+1}^{p} \frac{H(x, m) H(\xi, m)}{m^{2}}, \\
& \psi_{4}(x, \xi, p)=\frac{2 i}{b-a} Q_{3}(\xi) e^{\theta(x-a)} \sum_{m=M+1}^{p} \frac{\sin 2 m \pi\left(\frac{x-a}{b-a}\right)}{m} .
\end{aligned}
$$

Now sums of the type $\Sigma[(\sin m z) / m]$ as already noted remain uniformly bounded for all values of $z$. Since this is obviously true also of a sum $\Sigma\left[H(z, m) / m^{2}\right]$ we may conclude that

$$
|\varphi(x, \xi, p)|<A \text { ( a constant ), }
$$

for all $x$ and $\xi$ on $(a, b)$ and all $p$.

Let $\alpha$ and $\beta$ be chosen now as any two points of the interval $(a, b)$. We have then from (62) for $p>M$

$$
\int_{\alpha}^{\beta} \varphi(x, \xi, p) d \xi=\int_{\alpha}^{\beta} \varphi(x, \xi, M) d \xi+\sum_{i=1}^{4} \int_{\alpha}^{\beta} \psi_{i}(x, \xi, p) d \xi .
$$

It is our purpose to consider the convergence of this integral as $p \rightarrow \infty$. The first term on the right is free from $p$ and is clearly continuous over the interval $(a, b)$. It is likewise clear that the integral involving $\psi_{3}$ will converge uniformly to a function which is continuous on $(a, b)$. For the consideration of the remaining terms we recall the fact, also noted previously, that sums of the type $\Sigma[(\sin m z) / m]$ converge for all values of $z$ and do so uniformly except in the immediate neighborhood of $z=0, \pm 2 \pi, \cdots$. It follows because of the integration involved that the integrals containing $\psi_{1}$ and $\psi_{2}$ converge uniformly over the entire interval, and hence further because of the continuity of the individual terms that the limiting functions are continuous over $(a, b)$.

The integral containing $\psi_{4}$ is exceptional. The trigonometric sum involved remains in this case unaffected by the integration, and while this sum converges for all $x$ it does so uniformly only over an interval which does not extend to $x=a$, or $x=b$. Hence the integral converges uniformly 
for $a<a_{1} \leqq x \leqq b_{1}<b$, and the limiting function is continuous only on the open interval $a<x<b$. We may write, then, upon collecting our results,

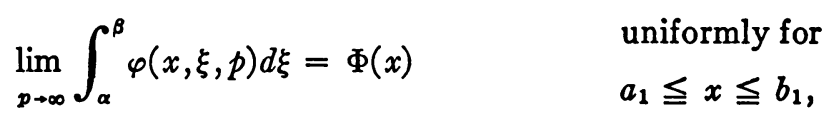

where $\Phi(x)$ is continuous for $a<x<b$.

45. The evaluation of $\Phi(x)$. Let $f(x)$ now be the function defined as follows:

$$
f(x)=\left\{\begin{array}{l}
1 \text { for } \alpha \leqq x \leqq \beta, \\
0 \text { for } a \leqq x<\alpha \text { and } \beta<x \leqq b .
\end{array}\right.
$$

This function satisfies the conditions of the theorem in $\S 43$ for expansibility in terms of the solutions $y_{m}(x)$, whence we have

$$
\sum_{m=-\infty}^{+\infty} \int_{a}^{b} f(\xi) z_{m}(\xi) d \xi \cdot y_{m}(x)=\bar{f}(x),
$$

where $\bar{f}(x)$ is bounded and differs from $f(x)$ only in the points $\alpha, \beta, a$ and $b$. Inasmuch as

$$
\int_{a}^{b} f(\xi) \varphi(x, \xi, p) d \xi=\int_{\alpha}^{\beta} \varphi(x, \xi, p) d \xi,
$$

we obtain with the use of (61) and (66) the result

$$
\Phi(x)=\sum_{m=-\infty}^{+\infty} \int_{a}^{b} f(\xi) v_{m}(\xi) d \xi \cdot u_{m}(x)-\bar{f}(x) .
$$

Multiplying this equality by $v_{l}(x)$ and integrating* it with respect to $x$ we find, since the set $u_{m}(x), v_{m}(x)$ is normalized biorthogonal, that

$$
\int_{a}^{b} \Phi(x) v_{l}(x) d x=\int_{a}^{b} f(\xi) v_{l}(\xi) d \xi-\int_{a}^{b} \bar{f}(x) v_{l}(x) d x=0 .
$$

Since this result may be derived for all values of $l$, whereas the set $u_{m}(x)$, $v_{m}(x)$ is closed, it follows that $\Phi(x) \equiv 0, a<x<b$. The relation (66) now takes the form

$$
\begin{aligned}
& \lim _{p \rightarrow \infty} \int_{\alpha}^{\beta} \varphi(x, \xi, p) d \xi=0 \\
& \text { uniformly for } \\
& a_{1} \leqq x \leqq b_{1},
\end{aligned}
$$

and this relation holds for all choices of the limits $\alpha$ and $\beta$ on $(a, b)$.

- From the character of the series as shown by the asymptotic forms of $u_{m}(x), v_{m}(x)$ this integration term by term is seen to be permissible. 
The properties (64) and (67), which have thus been established for the function $\varphi(x, \xi, p)$, are the hypotheses of a theorem by Lebesgue* in accordance with which it follows that if $f(x)$ is any function which is summable over the interval $(a, b)$ then

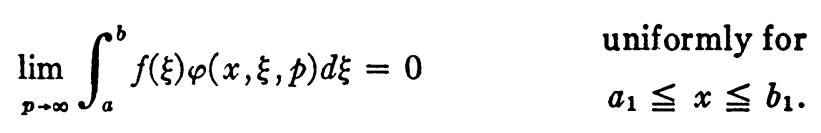

We defer the complete formulation of this result to the end of the chapter.

46. The evaluation of $\Phi(x)$ at the end points of $(a, b)$. It was observed in the derivation of (66) from (65) that the final term of (65) alone fails to converge to a function which is continuous over the entire closed interval $(a, b)$. Accordingly we have

$$
\begin{aligned}
\Phi(x)]_{x=l-}^{x=b} & =\left[\lim _{p \rightarrow \infty} \int_{\alpha}^{\beta} \psi_{4}(x, \xi, p) d \xi\right]_{x=b-}^{x=b} \\
& =\frac{2 i}{b-a} \int_{\alpha}^{\beta} Q_{3}(\xi) d \xi \cdot e^{\theta(b-a)}\left[\sum_{m=M+1}^{\infty} \frac{\sin 2 m \pi\left(\frac{x-a}{b-a}\right)}{m}\right]_{x=b-}^{x=b} .
\end{aligned}
$$

In virtue of the discussion just completed, however, $\Phi(b-)=0$. On the other hand the series on the right is, except for a finite number of continuous terms, the Fourier expansion of the function $\pi\left(\frac{1}{2}-(x-a) /(x-b)\right)$. The value of the bracket on the right is, therefore, readily found to be $\pi / 2$. Substituting, then, for $Q_{3}(\xi)$ its value as given by (53b), and for $e^{\theta(b-a)}$ its value $k_{1} / k_{2}$, we obtain the relation

$$
\Phi(b)=\frac{1}{2 k_{2}}\left(\nu k_{1}+\mu k_{2}\right) \int_{\alpha}^{\beta} \bar{\varphi}(\xi) d \xi,
$$

which reduces further because of (47) and (44b) to

$$
\Phi(b)=\int_{\alpha}^{\beta} \Omega_{2}(\xi) d \xi .
$$

The function $\Omega_{2}(\xi)$ involved here is that which occurs also in the characteristic equation (45). It is found in an entirely similar fashion that

$$
\Phi(a)=\int_{\alpha}^{\beta} \Omega_{1}(\xi) d \xi .
$$

* Annales de la Faculté des Sciences de Toulouse, ser. 3, vol. 1 (1909), p. 52 and p. 68. 
Let the functions $\varphi_{i}(\xi, p), i=1,2$, be defined now by the relations

$$
\begin{aligned}
& \varphi_{1}(\xi, p)=\varphi(a, \xi, p)-\Omega_{1}(\xi), \\
& \varphi_{2}(\xi, p)=\varphi(b, \xi, p)-\Omega_{2}(\xi) .
\end{aligned}
$$

It follows immediately from the bounded character of $\Omega_{1}(\xi)$ and $\Omega_{2}(\xi)$, and from the relations (64), (66) and (69) that

$$
\left|\varphi_{i}(\xi, p)\right|<A_{i} \quad(i=1,2)
$$

for all $\xi$ on $(a, b)$ and all $p$, and further that

$$
\lim _{p \rightarrow \infty} \int_{\alpha}^{\beta} \varphi_{i}(\xi, p) d \xi=0
$$

for all choices of $\alpha$ and $\beta$ on $(a, b)$. By Lebesgue's theorem, then, we may conclude that

$$
\lim _{p \rightarrow \infty} \int_{a}^{b} f(\xi) \varphi_{i}(\xi, p) d \xi=0
$$

for every function $f(x)$ which is summable over $(a, b)$.

47. The expansion theorems. If we observe now that

$$
\int_{a}^{b} f(\xi) \varphi(x, \xi, p) d \xi=\sum_{m=-p}^{p} \int_{a}^{b} f(\xi) v_{m}(\xi) d \xi \cdot u_{m}(x)-\sum_{m=-p}^{p} \int_{a}^{b} f(\xi) z_{m}(\xi) d \xi \cdot y_{m}(x),
$$

we may formulate of the results embodied in the relations (67) and (69) as follows.

THEOREM 10. If $f(x)$ is any function which is summable over the interval $(a, b)$, and if $F_{1 p}(x)$ and $F_{2 p}(x)$ are the corresponding sums

$$
\begin{aligned}
& F_{1 p}(x)=\sum_{m=-p}^{p} \int_{a}^{b} f(\xi) v_{m}(\xi) d \xi \cdot u_{m}(x), \\
& F_{2 p}(x)=\sum_{m=-p}^{p} \int_{a}^{b} f(\xi) z_{m}(\xi) d \xi \cdot y_{m}(x),
\end{aligned}
$$

where $u_{m}(x), v_{m}(x)$ are respectively the normalized solutions of the given integral equation and its associated equation, and $y_{m}(x), z_{m}(x)$ are respectively the normalized solutions of the related differential system (given by (60) above) and its adjoint system, then 


$$
\begin{aligned}
& \lim _{p \rightarrow \infty}\left[F_{1 p}(x)-F_{2 p}(x)\right]=0 \\
& \lim _{p \rightarrow \infty}\left[F_{1 p}(a)-F_{2 p}(a)\right]=\int_{a}^{b} f(\xi) \Omega_{1}(\xi) d \xi, \\
& \lim _{p \rightarrow \infty}\left[F_{1 p}(b)-F_{2 p}(b)\right]=\int_{a}^{b} f(\xi) \Omega_{2}(\xi) d \xi .
\end{aligned}
$$

The functions $\Omega_{1}(\xi)$ and $\Omega_{2}(\xi)$ are determined by the integral equation and are independent of the function $f(x)$.

A more explicit but less general formulation becomes possible if we utilize the known expansion theorem for the related differential system as given in $\$ 43$. Thus we may state

THEOREM 11. If $f(x)$ is any function which on the interval $(a, b)$ consists of at most a finite number of pieces, each real and continuous and having a continuous derivative, then $f(x)$ may be expanded in a series of the form

$$
f(x) \sim \sum_{m=-\infty}^{+\infty} \int_{a}^{b} f(\xi) v_{m}(\xi) d \xi \cdot u_{m}(x)
$$

This series converges to

to

$$
\frac{1}{2}\{f(x+)+f(x-)\} \quad \text { for } a<x<b \text {; }
$$

$$
\frac{1}{2 k_{1}}\left\{k_{1} f(a+)+k_{2} f(b-)\right\}+\int_{a}^{b} f(\xi) \Omega_{1}(\xi) d \xi \quad \text { for } x=a \text {; }
$$

to

$$
\frac{1}{2 k_{2}}\left\{k_{1} f(a+)+k_{2} f(b-)\right\}+\int_{a}^{b} f(\xi) \Omega_{2}(\xi) d \xi \quad \text { for } x=b .
$$

Appendix. AN EXAMple

It is of interest to apply the preceding theory to a specific example particularly because of the fact that the theory demands in Chapters 8 and 11 that the ratio $k_{1} / k_{2}$ and the functions $\Omega_{1}(x)$ and $\Omega_{2}(x)$ be independent of the parameters $\mu$ and $\nu$. We consider therefore the integral equation

$$
u(x)=\lambda \int_{0}^{b} K(x, \xi) u(\xi) d \xi,
$$

where

$$
K(x, \xi)= \begin{cases}x^{2} \xi^{2}+2 & \text { for } x \geqq \xi \\ x^{2} \xi^{2}+1 & \text { for } x<\xi\end{cases}
$$


The equation is in normal form, and computing the various functions by the respective formulas we find readily

$$
\begin{aligned}
\Delta & =\mu^{2}+\left(b^{4}+3\right) \mu \nu+2 \nu^{2}, \\
W(x) & =2 \nu+\mu\left(b^{2} x^{2}+1\right), \\
V(\xi) & =\mu+\nu\left(b^{2} \xi^{2}+2\right), \\
E(x, \xi) & =\frac{2(\mu+2 \nu)}{\Delta}\left[(\mu+\nu) x^{2}-\nu b^{2}\right] \xi, \\
D & =\frac{(\mu+\nu)\left[\left(2-b^{4}\right) \mu+\left(4+2 b^{4}\right) \nu\right]}{2 \Delta}, \\
F(x, \xi) & =\frac{-E(x, \xi)}{D}, \\
f(x, \xi) & =\frac{-8(\mu+2 \nu) x \xi}{\left(2-b^{4}\right) \mu+\left(4+2 b^{4}\right) \nu}, \\
\varphi(x) & =\frac{8 b^{2} x}{\left(2-b^{4}\right) \mu+\left(4+2 b^{4}\right) \nu}, \\
\int_{0}^{b} V(\xi) \varphi(\xi) d \xi & =\frac{2 b^{4}\left[2 \mu+\left(4+b^{4}\right) \nu\right]}{\left(2-b^{4}\right) \mu+\left(4+2 b^{4}\right) \nu}, \\
k_{1} & =\frac{\left(2+b^{4}\right)(\mu+\nu)}{D}, \\
k_{2} & =\frac{\left(2-b^{4}\right)(\mu+\nu)}{2 D} . \\
& =\frac{(2 D}{D},
\end{aligned}
$$

The ratio $k_{1} / k_{2}$ is thus free from $\mu, \nu$, having the value

Further we find

$$
\frac{k_{1}}{k_{2}}=\frac{2\left(2+b^{4}\right)}{2-b^{4}} \text {. }
$$

whence

$$
V^{\prime}(x)+\int_{0}^{b} V(t) f(t, x) d t=\frac{-2 b^{2} x(\mu+\nu)}{D}
$$

$$
\begin{aligned}
& \Omega_{1}(x)=\frac{b^{2} x}{2+b^{4}} \\
& \Omega_{2}(x)=\frac{-2 b^{2} x}{2-b^{4}} .
\end{aligned}
$$

These values are thus also free from $\mu$ and $\nu$ as they should be.

Dartmouth College,

HANOVER, N. H. 